\title{
Article \\ NAC Transcription Factor PwNAC11 Activates ERD1 by Interaction with ABF3 and DREB2A to Enhance Drought Tolerance in Transgenic Arabidopsis
}

\author{
Mingxin $\mathrm{Yu}^{\dagger}{ }^{\dagger}$ Junling Liu ${ }^{\dagger}$, Bingshuai Du, Mengjuan Zhang, Aibin Wang and Lingyun Zhang *(D) \\ Key Laboratory of Forest Silviculture and Conservation of the Ministry of Education, The College of Forestry, \\ Beijing Forestry University, Beijing 100083, China; ymxbjfu@163.com (M.Y.); liujunling022@163.com (J.L.); \\ dubingshuai624@163.com (B.D.); liujunling02@126.com (M.Z.); wangaibin126@126.com (A.W.) \\ * Correspondence: lyzhang@bjfu.edu.cn; Tel.: +86-10-6233-6044 \\ + These authors contributed equally to this work.
}

check for updates

Citation: Yu, M.; Liu, J.; Du, B.; Zhang, M.; Wang, A.; Zhang, L. NAC Transcription Factor PwNAC11 Activates ERD1 by Interaction with ABF3 and DREB2A to Enhance Drought Tolerance in Transgenic Arabidopsis. Int. J. Mol. Sci. 2021, 22, 6952. https://doi.org/10.3390/ ijms22136952

Academic Editor: Víctor Quesada

Received: 31 May 2021

Accepted: 22 June 2021

Published: 28 June 2021

Publisher's Note: MDPI stays neutral with regard to jurisdictional claims in published maps and institutional affiliations.

Copyright: (c) 2021 by the authors. Licensee MDPI, Basel, Switzerland. This article is an open access article distributed under the terms and conditions of the Creative Commons Attribution (CC BY) license (https:// creativecommons.org/licenses/by/ $4.0 /)$.
Abstract: NAC (NAM, ATAF1/2, and CUC2) transcription factors are ubiquitously distributed in eukaryotes and play significant roles in stress response. However, the functional verifications of NACs in Picea (P.) wilsonii remain largely uncharacterized. Here, we identified the NAC transcription factor PwNAC11 as a mediator of drought stress, which was significantly upregulated in P. wilsonii under drought and abscisic acid (ABA) treatments. Yeast two-hybrid assays showed that both the full length and C-terminal of PwNAC11 had transcriptional activation activity and PwNAC11 protein cannot form a homodimer by itself. Subcellular observation demonstrated that PwNAC11 protein was located in nucleus. The overexpression of PwNAC11 in Arabidopsis obviously improved the tolerance to drought stress but delayed flowering time under nonstress conditions. The steady-state level of antioxidant enzymes' activities and light energy conversion efficiency were significantly increased in PwNAC11 transgenic lines under dehydration compared to wild plants. PwNAC11 transgenic lines showed hypersensitivity to ABA and PwNAC11 activated the expression of the downstream gene ERD1 by binding to ABA-responsive elements (ABREs) instead of drought-responsive elements (DREs). Genetic evidence demonstrated that PwNAC11 physically interacted with an ABA-induced protein-ABRE Binding Factor3 (ABF3) - and promoted the activation of ERD1 promoter, which implied an ABA-dependent signaling cascade controlled by PwNAC11. In addition, qRT-PCR and yeast assays showed that an ABA-independent gene-DREB2A-was also probably involved in PwNAC11-mediated drought stress response. Taken together, our results provide the evidence that PwNAC11 plays a dominant role in plants positively responding to early drought stress and ABF3 and DREB2A synergistically regulate the expression of ERD1.

Keywords: Picea wilsonii; transcription factor; PwNAC11; drought stress; ABA signaling

\section{Introduction}

Water deficit is one of the most disruptive abiotic stresses influencing plant growth and development [1,2]. As environments of plants have gradually deteriorated as a result of global warming, it is of great significance to understand the molecular mechanisms of plants themselves responding to abiotic stress, especially drought stress, for further cultivating new varieties with strong adaptability. In order to fight against abiotic stress, plants have evolved a series of sophisticated but effective strategies to protect themselves from the environmental disadvantages, including stress escape and stress tolerance [3,4]. Stress escape mainly occurs under mild stress conditions, which allows plants to accelerate the growth and flowering processes in order to avoid prematurely dying of water deprivation. Stress tolerance is a regulatory mechanism by which plants can endure long-term stress and maintain their vitality under such environmental pressure [5]. 
Particularly, transcription factors (TFs) play a vital role in stress regulatory mechanisms by selectively binding to cis-elements in the promoter of downstream genes [6]. NACs are one of the most widely reported families of transcription factors in plants, which can be divided into NAM, ATAF and CUC subfamilies according to their interspecific DNAbinding domains. The N-terminal domain of NAC TF can be divided into five conserved subdomains (A, B, C, D, and E). Among them, the A domain is related to the formation of dimers, the $\mathrm{C}$ and $\mathrm{D}$ domains are highly conserved with binding affinity to promoters, but the $\mathrm{B}$ and $\mathrm{E}$ domains are relatively non-conserved. By comparison, the $\mathrm{C}$-terminal exhibits more structural variability and is considered as the transcription activation domain [7]. Ooka et al. conducted a comprehensive analysis of 180 NAC family proteins in Arabidopsis and rice for the first time [8]. According to the similarity of the NAC domain sequences between Arabidopsis and rice, the 180 proteins were divided into two large groups and 18 subgroups. A growing body of evidence has shown that NAC transcription factors are widely involved in plant response to abiotic/biotic stress [9], senescence [10], fruit ripening [11] and hormonal regulation [12], etc.

Over the years, a variety of NAC TFs in different plant species have been reported to play positive roles in response to adverse environments. For example, three NAC transcription factors-ANAC019, ANAC055 and ANAC072-were substantially induced by drought, salt and ABA treatments, the overexpression of which improved the drought tolerance of transgenic Arabidopsis [13]. Similarly, the overexpression of pepper CaNAC46 in Arabidopsis significantly inhibited ROS accumulation and the transgenic lines are less susceptible to salt stress [14]. Similarly, TaSNAC8-6A overexpression in wheat exhibited increased drought stress tolerance and this process was proven to be controlled by the auxin- and drought response pathways by transcriptomic analysis [15]. The NAC family member JUB1 was proven to be a regulator of drought stress, and the overexpression of tomato SIJUB1 or Arabidopsis AtJUB1 could increase drought tolerance in transgenic tomato by directly binding to the promoters of SIDELLA, SIDREB2 and SIDREB1 [16], suggesting that the regulation mechanisms of the NAC transcription factors involved in abiotic stress are relatively specific among different species. In woody plants, BpNAC012 derived from Betula platyphylla activated the core CGTG/A elements to promote the expression of stressresponsive genes, which further enhanced osmotic and salt stress tolerance in BpNAC012 transgenic birch [17]. In addition, many of the NAC TFs were demonstrated to regulate abscisic acid (ABA)-induced stress response process. ONAC022 showed transcriptional abundance after $\mathrm{ABA}$ and drought treatments in rice and NAC022 OE lines improved sensitivity to exogenous ABA [18]. ANAC096 could directly interact with ABF2 and ABF4 to activate the expression of $R D 29 A$, thus participating in dehydration and osmotic stresses regulation in an ABA-dependent pathway in Arabidopsis [19]. Although the functions of the NAC TF family have been investigated in various plant species, it has been difficulties of in-depth study for revealing the complicated and efficient molecular mechanisms inside.

$\mathrm{ABA}$, an important hormone upstream of transcription factors, acts as a messenger in the biological processes of stomatal conductance, seed dormancy and germination, leaf senescence, and stress response [20-22]. Once suffering biotic or abiotic stress, the content of ABA shows a notable increase, and endogenous $\mathrm{ABA}$ will be transported to the ground part through the transmission of xylem to reduce stomatal conductance, further reducing water loss and activating the corresponding receptors to initiate signal transduction in response to stress [23]. ABA synthesis in plants is mainly formed through the degradation of carotenoids: several crucial enzymatic proteins, such as NCED, ABA2 and AAO, are involved in this process [24]. The transduction of the ABA signal mainly consists of three key ABA receptors, PP2C, PYP/PYL/RCAR, and SNRK2 kinase [25]. In the presence of ABA, SnRK2 could phosphorylate downstream ABA-responsive element (ABRE)-binding factors (ABFs). Meanwhile, some transcription factors such as NAC, bZIP, MYB and WRKY, are reported to activate the expression of ABA-dependent genes through binding to cisacting elements on promoters of downstream genes containing specific ABRE motifs. In addition, other cis-acting regulatory elements, such as dehydration responsive elements 
(DREs) are also demonstrated to respond to external stresses in an ABA-independent pathway [26,27].

Although the roles of NAC transcription factors involved in abiotic stress response have been extensively explored, the knowledge regarding NAC TFs participating in the regulation of coniferous forests responding to abiotic stress is still limited. Picea wilsonii, an endemic species of coniferous forests, which is best characterized by tolerance to abiotic stress and environment adaptability, is widespread in northern China. Picea wilsonii also has ornamental value in landscaping based on its impressing appearance and lush green crown [28]. Given its advantageous characteristics, it is of great significance to screen and reveal the molecular mechanisms of its abiotic resistance. Previously, we identified two NAC transcription factors-PwNAC2 and PwNAC30-from P. wilsonii, which acted as positive and negative regulators in response to abiotic stress, respectively $[28,29]$, suggesting the complexity and functional diversity of NAC TF members in coniferous forests. Here, based on the high-throughput RNA-sequencing (RNA-seq) of the expression profiles of P. wilsonii in the absence or presence of drought treatments [30], we identify PwNAC11 as one of the most differentially expressed genes (DEGs) on a transcriptional level and further elucidate the function and molecular mechanism underlying the ABA-mediated drought stress response. We found that overexpression of PwNAC11 in Arabidopsis obviously improved tolerance to drought stress by binding to the promoter of ERD1. During this process, the interaction of PwNAC11 with ABF3 synergistically activated the expression of the ERD1 promoter, resulting in increased resistance to drought stress. The biochemical and physiological evidence showed that ABA positively promoted the regulation of the PwNAC11-mediated abiotic stress response. Our study provides the basis for a new understanding of NAC TF in genetic breeding and the improvement of coniferous forest germplasm resources.

\section{Results}

\subsection{Bioinformatics Analysis of PwNAC11}

PwNAC11 was cloned from cDNA library of $P$. wilsonii. It has an open reading frame with a length of $954 \mathrm{bp}$ and encodes a protein with 317 amino acid. Protein multiple sequence alignment and BLAST analysis showed that the PwNAC11 protein had a typical NAM (no apical meristem) domain without transmembrane domain (TMD) (Figure 1A). Phylogenetic analysis indicated that PwNAC11 was close to the homology of Picea sitchensis (Figure 1B).

To determine whether PwNAC11 potentially functions as a transcription factor, we first examined the subcellular localization of the PwNAC11 protein. The results showed that the fluorescent signals for the empty vector were widely detected in the nucleus, cell membrane, and cytoplasm, but the PwNAC11-GFP fusion protein was only detected in the nucleus, indicating that PwNAC11 is a nucleus-located transcription factor (Figure 1C). The yeast two-hybrid assay was conducted to detect whether PwNAC11 had transcriptional activating activity. We found that only the yeast cells containing pGBKT7-PwNAC11, pGBKT7PwNAC11-C, and pGBKT7-ANAC092 grew well on SD/Trp-His-Ade selective medium, suggesting that both full length and C-terminal of PwNAC11 have transcriptional activating activity (Figure 1D). Moreover, yeast strains AH109 co-transformed with pGBKT7PwNAC11 + pGADT7-PwNAC11 and pGBKT7-PwNAC11 $\triangle C$ + pGADT7-PwNAC11 cannot grow on SD/Trp-Leu-His-Ade selective medium, indicating that PwNAC11 cannot form a homodimer by itself (Figure 1E).

To elucidate the response of PwNAC11 to drought stress, qRT-PCR was performed with 4-week-old P. wilsonii seedlings. The results showed that the expression of PwNAC11 was dramatically induced at $3 \mathrm{~h}$ by PEG or ABA treatment, indicating that PwNAC11 is probably involved in the process of plants' early responses to adverse environments (Figure 1F). 
A
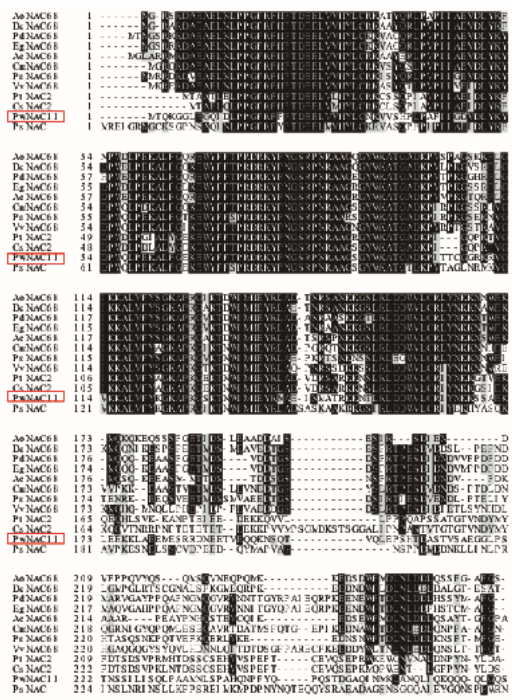

$\mathrm{B}$

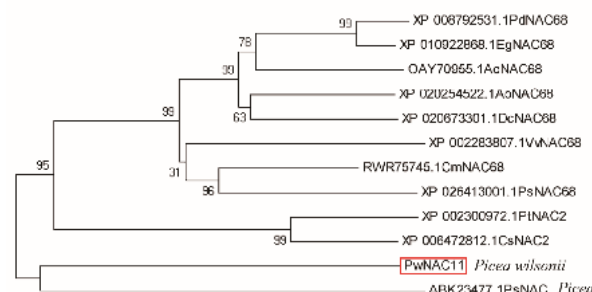

$\longmapsto$
C
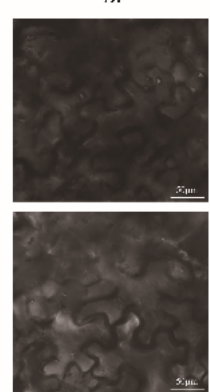

D

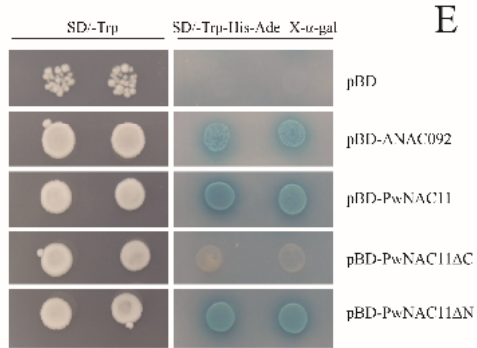

$\mathrm{F}$

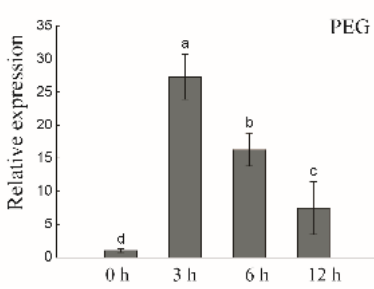

GiFP

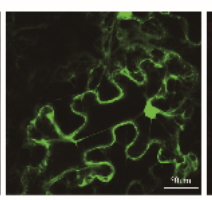

$\mathrm{E}$
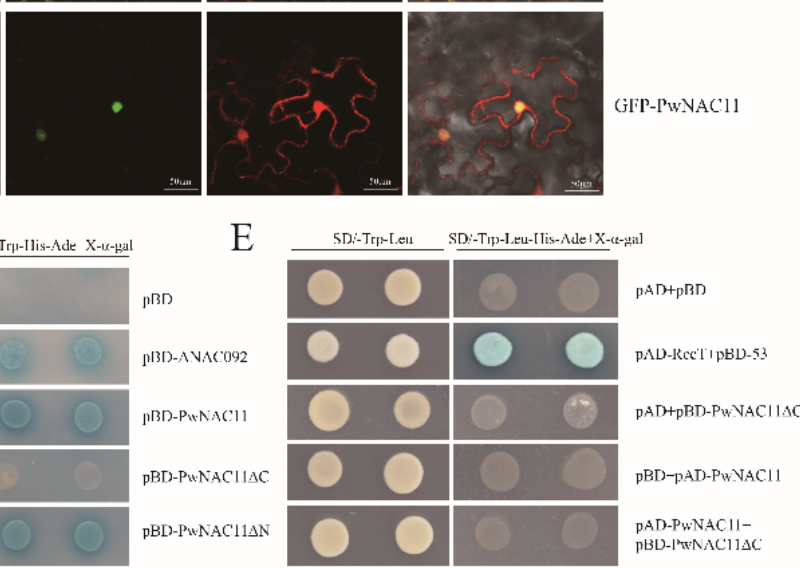

$\mathrm{ABA}$

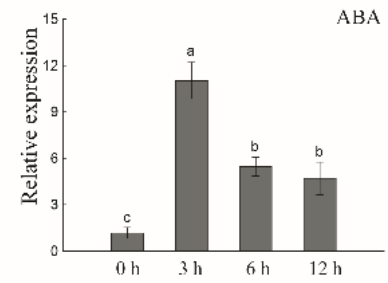

Figure 1. (A) Protein multiple sequence alignment of PwNAC11. (B) Phylogenetic analysis of PwNAC11. Numbers on each branch represent the confidence of 1000 repetitions. (C) Subcellular localization of PwNAC11. RACK1A-RFP is used as a marker gene which locates in nucleus, cytoplasm and cell membrane. The bar in each picture is $10 \mu \mathrm{m}$. (D) Yeast trans-acting activity assay. pBD-ANAC092 was used as positive control. pBD-PwNAC11 $\triangle \mathrm{N}$ refers to the PwNAC11 protein without N terminal (171-318) and pBD-PwNAC11 $\triangle$ C refers to the PwNAC11 protein without C terminal (1-153). (E) Yeast two-hybrid assay for PwNAC11 forming homodimers. pAD-RecT+pBD-53 was used as positive control and pAD + pBD was used for negative control. (F) Expression profiles of PwNAC11 in P. wilsonii under drought and ABA treatments. Different letters indicate the significant differences at $p<0.05$.

\subsection{Overexpression of PwNAC11 Enhances Drought Tolerance in Transgenic A. thaliana}

In order to investigate the function of PwNAC11 in response to drought stress, we heterogeneously expressed PwNAC11 in Arabidopsis under the control of CaMV 35 S promoter. Two T3 lines with PwNAC11 highly expressed, named OE2 and OE3, were selected for further functional analysis (Figure S1). The seeds of wild type (WT), empty vector (VC) and overexpression (OE2 and OE3) lines were sown on MS medium with selected concentrations of mannitol. It was found that there was no significant difference in the germination rates among these lines under normal conditions. Under the simulated drought conditions, the growth of the WT and VC groups were severely inhibited, whereas the germination rates of the seeds of the two OE lines were less affected (Figure 2A). For example, the germination percentage of $\mathrm{OE}$ lines were over $80 \%$ under mannitol conditions of $200 \mathrm{mM}$ compared to less than $60 \%$ of the WT and VC lines (Figure $2 \mathrm{~F}, \mathrm{H}$ ). Moreover, the PwNAC11 transgenic lines showed stronger germination rates. Under $100 \mathrm{mM}$ mannitol treatment, the germination rates of $\mathrm{OE}$ lines were 1.6 times higher than control groups on the 4th day. Meanwhile, in the presence of $300 \mathrm{mM}$ mannitol, the germination rate of OE3 line was nearly $20 \%$ on the 3rd day, but the seeds of control groups showed almost no germination at the same time, implying that the $\mathrm{OE}$ lines had stronger seed vitality at the early stage of germination under drought treatment. Similar results were found in the root length 
experiment, especially under the treatment of $200 \mathrm{mM}$ mannitol, in which the average root length of the OE lines was $10 \mathrm{~mm}$ longer than that of WT and VC groups (Figure 2B,C).

A

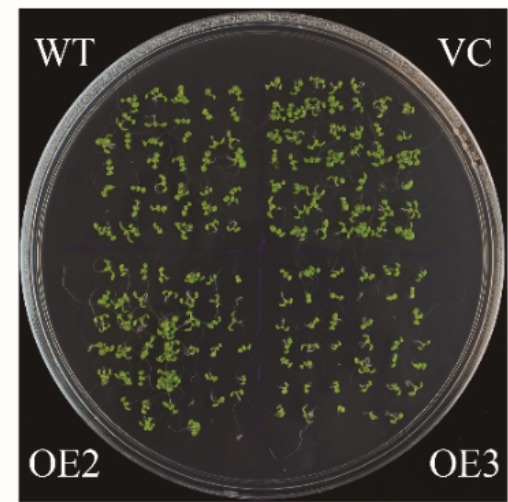

Normal

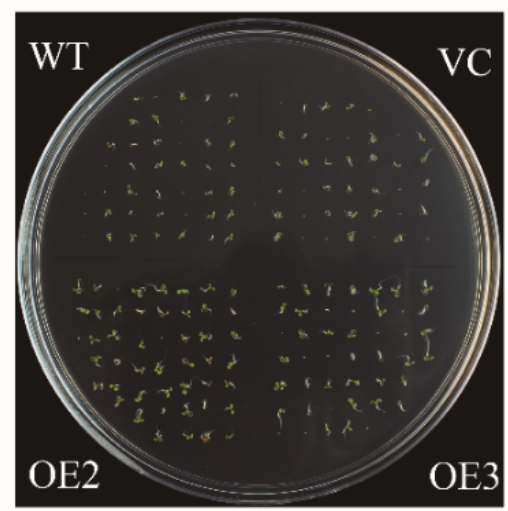

$200 \mathrm{mM}$ Mannitol

B

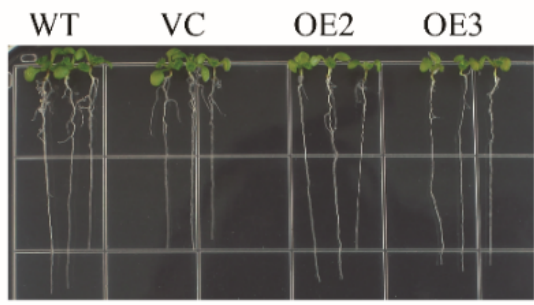

Normal

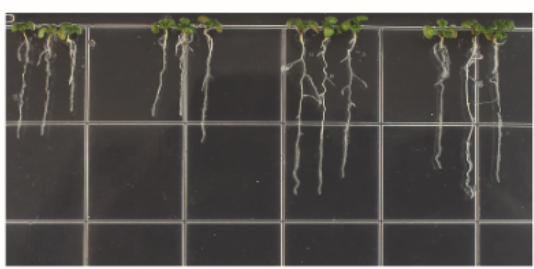

$200 \mathrm{mM}$ Mannitol

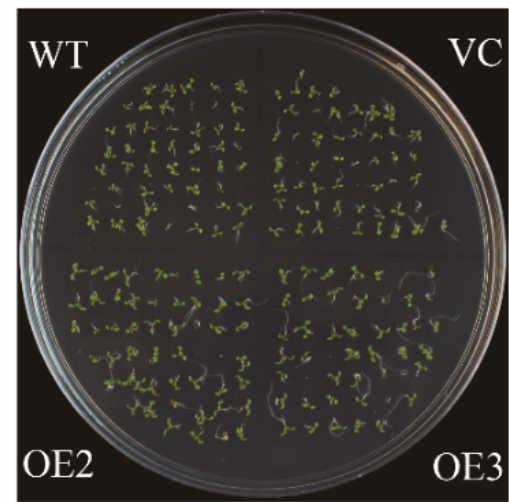

$100 \mathrm{mM}$ Mannitol

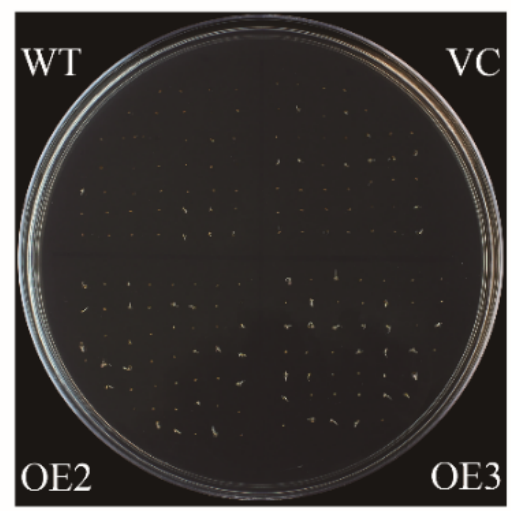

$300 \mathrm{mM}$ Mannitol

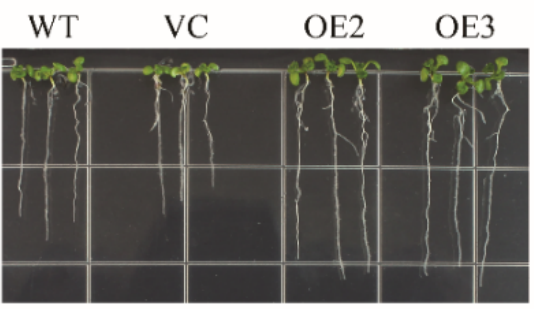

$100 \mathrm{mM}$ Mannitol

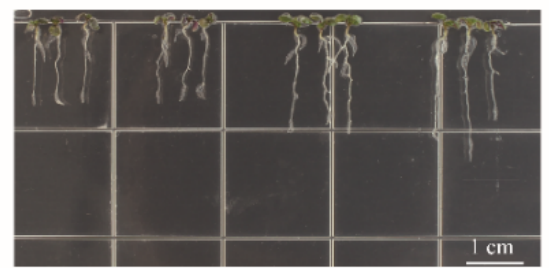

$300 \mathrm{mM}$ Mannitol
$\mathrm{C}$
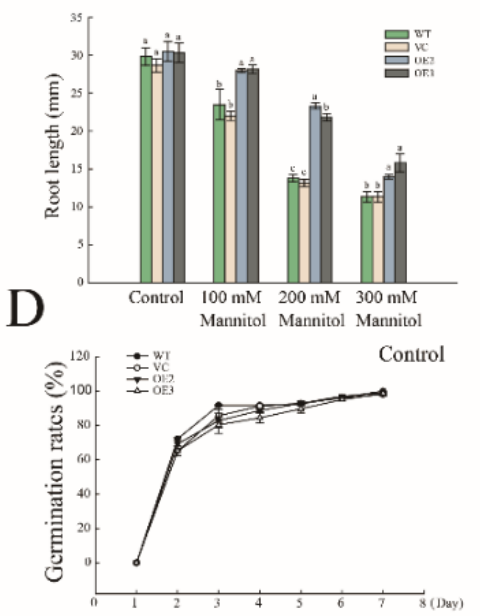

$\mathrm{E}$

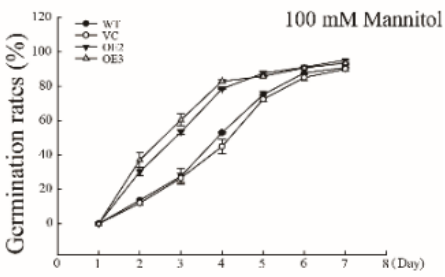

$\mathrm{F}$

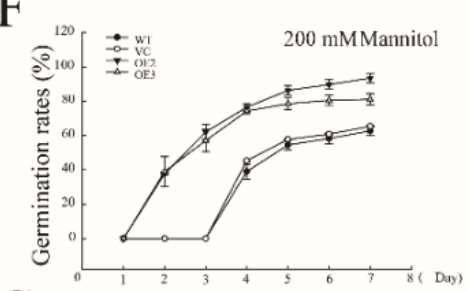

$\mathrm{G}$

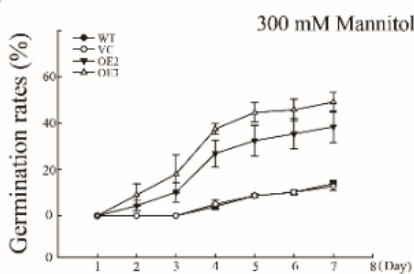

$\mathrm{H}$

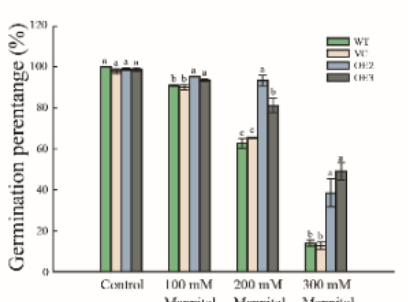

Figure 2. PwNAC11 overexpression promotes seed germination under simulated drought conditions. (A) Germination assay under different concentrations of mannitol treatments. (B) Root elongation assays under different concentrations of mannitol treatments. (C) Quantification of root length under different concentrations of mannitol treatments. Different letters indicate significant differences at $p$-value $<0.05$. (D-G) Germination rates of different lines under different concentrations of mannitol treatments within 7 days. (H) Germination percentage of each line after 7-day simulated drought treatment. Different letters indicate significant differences at $p$-value $<0.05$. 
To analyze the role of PwNAC11 in drought stress during adult stage, 4-week-old seedlings were exposed to drought treatment. We found that the transgenic lines only wilted slightly and still maintained a normal growth state with a final survival percentage of $74 \%$ after drought treatment and followed by re-watering (Figure 3C), but the leaves of most of the WT and VC plants were wilted seriously or even dead, which were unable to recover after re-watering (Figure 3A). We also counted the relative water content (RWC) of each line. The RWC of the OE lines was approximately $50 \%$, while it was only $25 \%$ for WT and $\mathrm{VC}$ lines (Figure 3B).

A
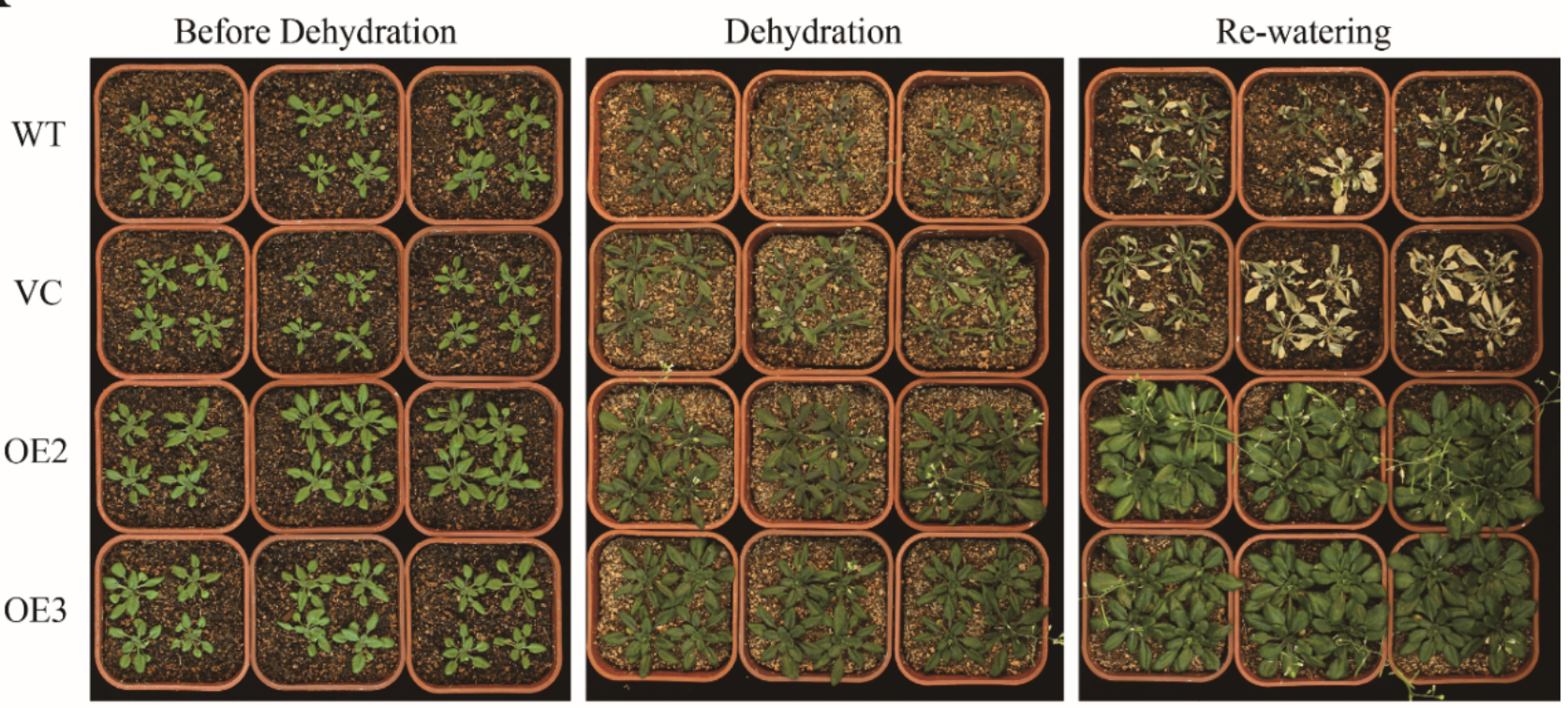

B
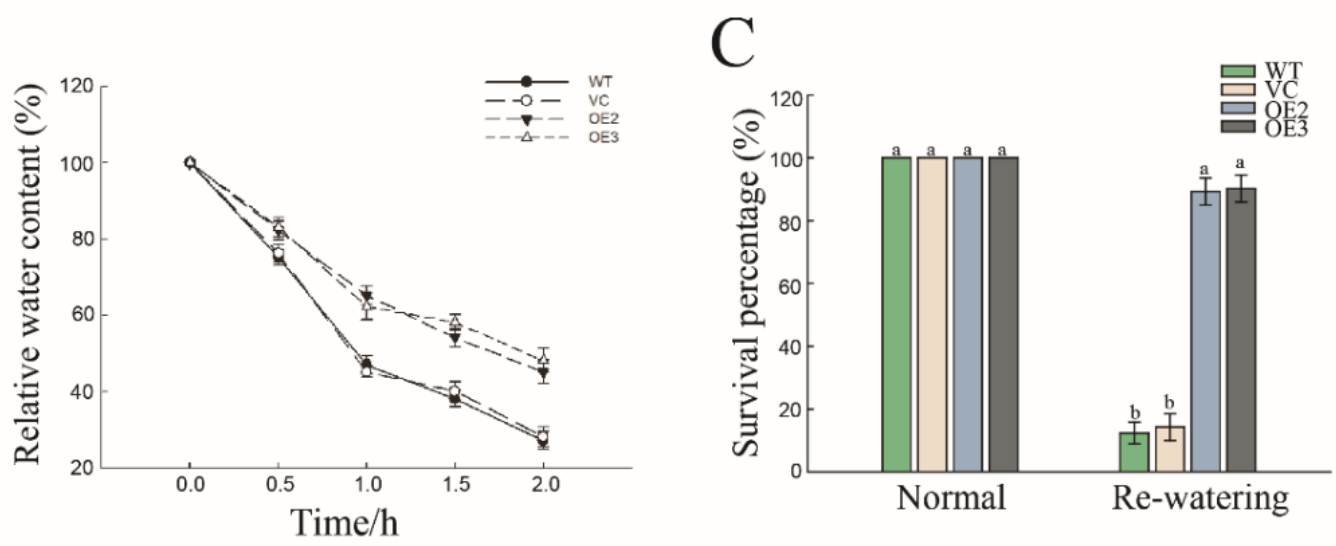

Figure 3. Overexpression of PwNAC11 improves tolerance to drought stress. (A) Phenotypes of different lines under drought conditions. Dehydration lasted 14 days, followed by re-watering for 3 days. (B) Relative Water Content (RWC) after drought stress. Isolated leaves were placed at room temperature and dehydrated for $2 \mathrm{~h}$. (C) Survival percentage before drought treatment and after re-watering. Different letters indicate the significant differences at $p<0.05$.

\subsection{PwNAC11 Is Involved in the Regulation of ROS Accumulation and Photosynthetic Efficiency under Drought Conditions \\ Drought stress has been reported to accelerate the accumulation of ROS. In order to investigate whether PwNAC11 promotes the degradation of superfluous hydrogen peroxide and superoxide in the leaves, we conducted the DAB and NBT staining. It was found that the leaves of each line were hardly stained under normal conditions. Nevertheless, once submitted to drought stress, the staining intensity of the leaves for WT and VC groups was substantially deeper than those of OE lines (Figure 4A,B), suggesting}


more ROS accumulated in WT and VC groups compared with OE lines. Further antioxidant enzymes activity assay indicated the activities of SOD and CAT increased apparently in two OE lines during drought treatments compared to normal conditions (Figure 4C,D). The severity of lipid oxidation was reflected in the MDA content. Upon drought treatment, the content of MDA in the OE lines was below $80 \mathrm{nmol} / \mathrm{mg}$, which was $37 \%$ lower than that in WT and VC lines (Figure 4E). These results indicate that overexpression of PwNAC11 significantly improved the scavenging abilities of ROS and contributed to the protection from peroxidation damage under drought stress. In addition, the decomposition rate of chlorophyll was reduced in transgenic lines (Figure 4F), suggesting that PwNAC11 probably participates in the regulation of the photosynthetic system.
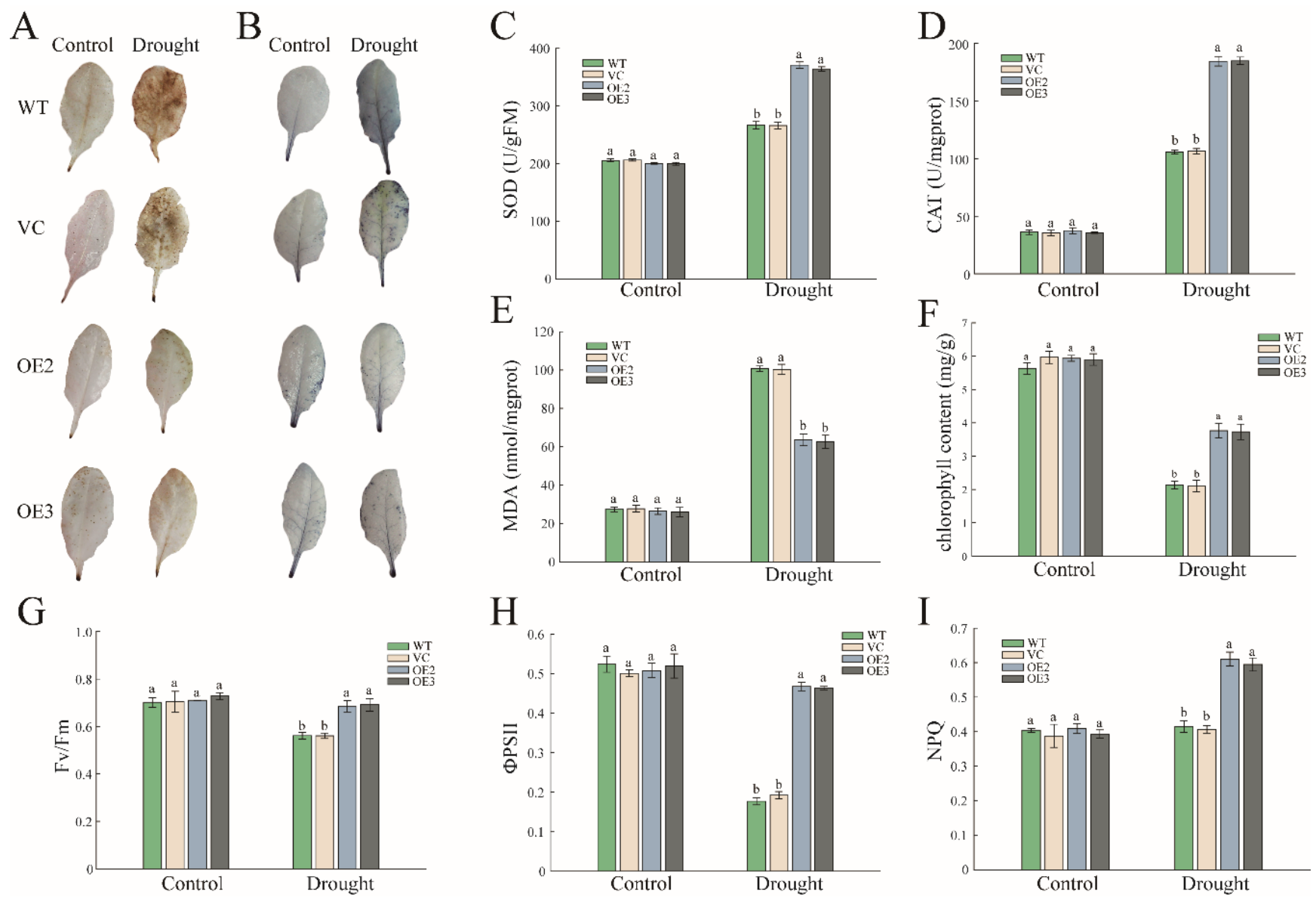

Figure 4. Overexpression of PwNAC11 enhances ROS scavenging ability in transgenic Arabidopsis. (A) Histochemical detection of hydrogen peroxide using DAB staining. (B) Histochemical detection of superoxide using NBT staining. (C) The activity of SOD. (D) The activity of CAT. (E) The content of MDA. (F) The content of chlorophyll. (G) Measurement of Fv/Fm under normal and drought conditions. (H) Measurement of ФPSII under normal and drought conditions. (I) Measurement of NPQ under normal and drought conditions. Different letters indicate significant differences at $p$-value $<0.05$.

Drought stress may also reduce the photosynthetic rate, leading to the decline in the efficiency of photosynthesis ( $\mathrm{Fv} / \mathrm{Fm}$ ) and quantum yield of PSII electron transport (ФPSII). In order to verify whether PwNAC11 is involved in the protection of plant photosynthesis under drought stress, several photosynthetic parameters were subsequently measured after 14 days of drought stress. It was observed that Fv /Fm fell by $22 \%$ in WT and VC after exposure to drought stress, but it was almost unchanged in the two OE lines (Figure 4G). Similarly, the $\Phi P S I I$ decreased significantly by $65.4 \%$ in WT and only by $10 \%$ in OE lines (Figure 4H). On the contrary, nonphotochemical quenching (NPQ) increased dramatically in the $\mathrm{OE}$ lines under drought treatment and showed no significant difference in the WT 
and VC groups in the absence or presence of drought treatment (Figure 4I), suggesting that PwNAC11 confers increased photoprotection in OE lines.

\subsection{Overexpression of PwNAC11 Activates the Expression of Stress-Responsive Genes}

Our study found that overexpression of PwNAC11 improved plant resistance to drought stress and can be greatly induced by ABA. To further quantify the expression levels of stress-responsive and ABA-induced genes of different lines, we selected such genes as ANAC019, ANAC055, DREB2A, ERD1, ATHB-7 and ABF3 for qRT-PCR analysis based on the reports on the regulation network of homologous genes of PwNAC11 in Arabidopsis (Figure 5). Under simulated drought conditions, most of these genes showed higher expression levels in OE lines compared with the WT and VC groups. ANAC055 and $E R D 1$ were even upregulated in normal conditions compared with WT and VC groups (Figure 5C,E). For ERD1, the expression was 12 times higher in $\mathrm{OE}$ lines than that in the other groups under PEG treatment, and $A T H B-7$ showed more than a twenty-fold increase when subjected to $12 \mathrm{~h}$ PEG treatment (Figure 5F). These results suggested that PwNAC11 promoted the expression of stress-related and ABA-responsive genes, and thus, improved the stress tolerance in the plants.

A

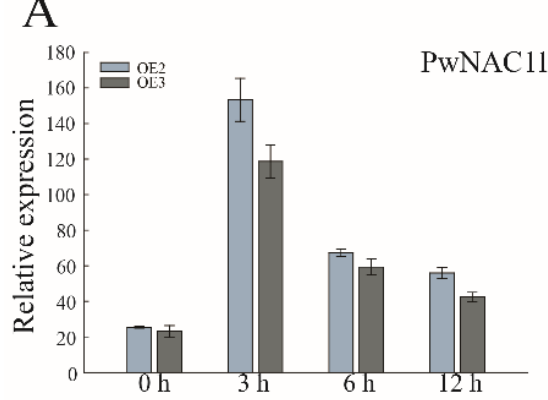

$\mathrm{D}$

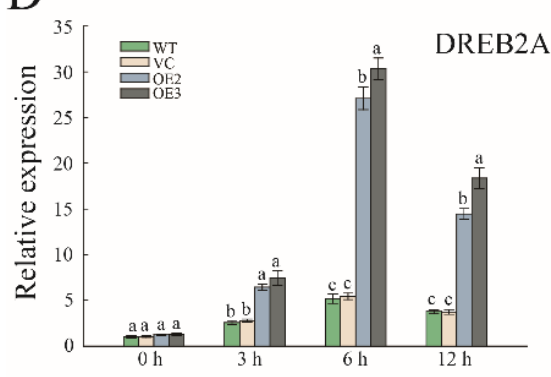

$\mathrm{G}$

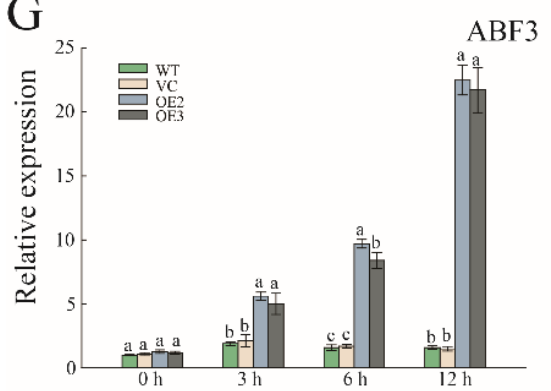

$\mathrm{B}$

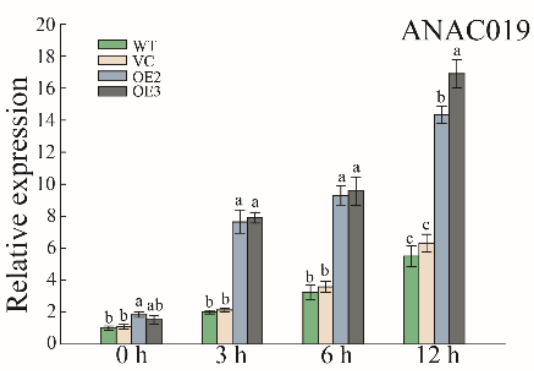

$\mathrm{E}$

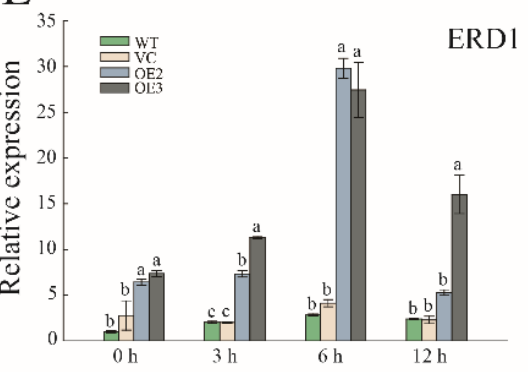

$\mathrm{H}$

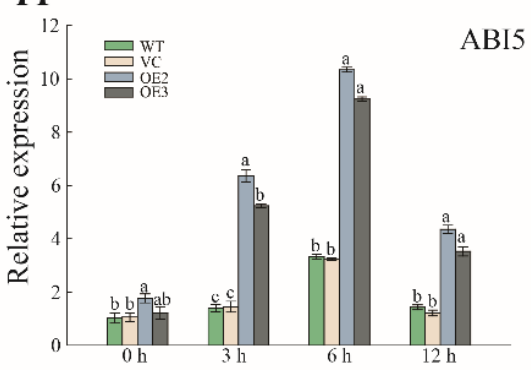

C

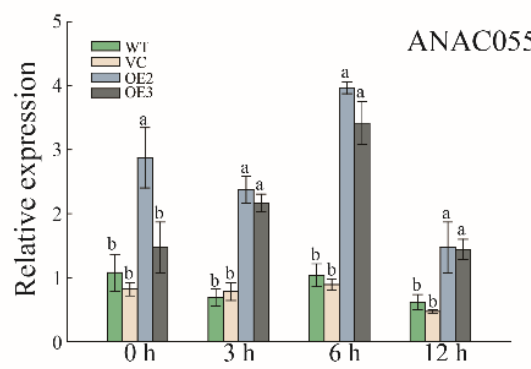

F

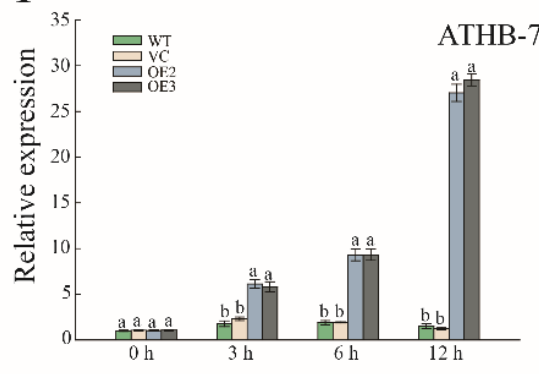

I

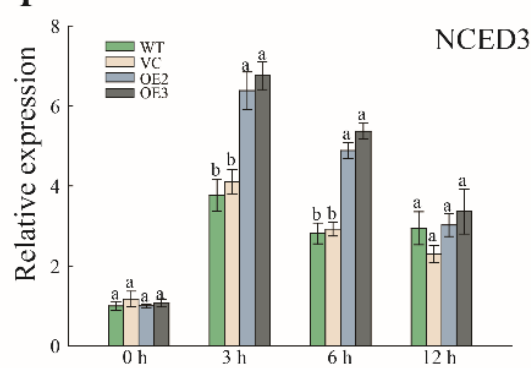

Figure 5. Expression profiles of some stress-responsive genes and ABA-responsive genes after PEG treatment. (A-I) Expression profiles of these genes in OE, WT, and VC lines under simulated drought conditions. The data are represented as means \pm standard error from three independent replications. Different letters indicate significant differences at $p$-value $<0.05$. 


\subsection{Overexpression of PwNAC11 Increases ABA Sensitivity and Promotes ABA-Induced Stomatal Closure in A. thaliana}

Since the expression of PwNAC11 was upregulated under exogenous ABA treatment in P. wilsonii, we speculate that PwNAC11 is responsive to the ABA signal. To prove this hypothesis, we conducted another phenotypic experiment by exposing the seeds of each line to different concentrations of exogenous ABA to test the response of PwNAC11 to the ABA signal. We found that the germination percentage of OE2 and OE3 decreased to $70 \%$ under $1 \mu \mathrm{M}$ ABA treatment, while it was maintained at nearly $80 \%$ in the WT and VC lines (Figure $6 \mathrm{~A}, \mathrm{C}$ ). The results of the root length experiment also proved that the root length of the OE lines was almost $5 \mathrm{~mm}$ shorter than that of the WT and VC lines, whether under $0.5 \mu \mathrm{M}$ or $1 \mu \mathrm{M}$ ABA treatment (Figure $6 \mathrm{~B}, \mathrm{D}$ ).
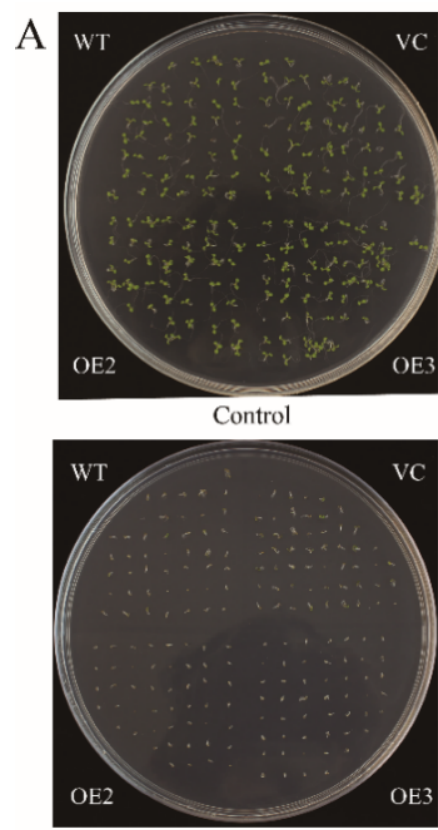

$0.5 \mu \mathrm{M} \mathrm{ABA}$

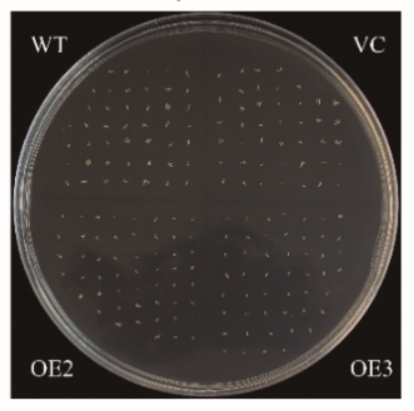

$1 \mu \mathrm{M} \mathrm{ABA}$
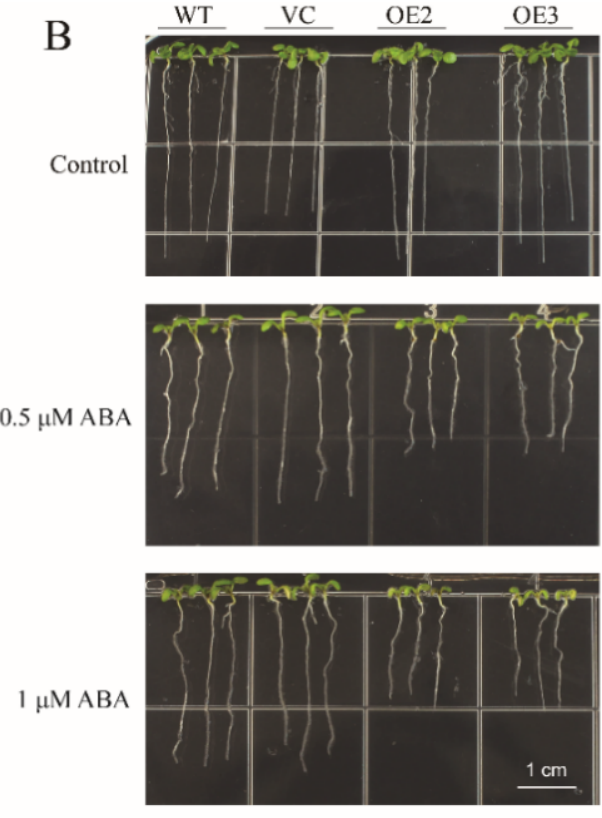

F

WT
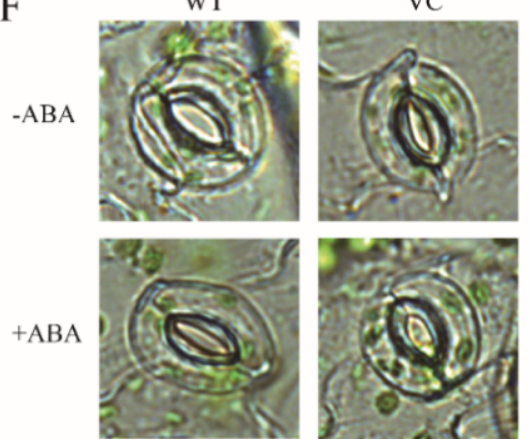

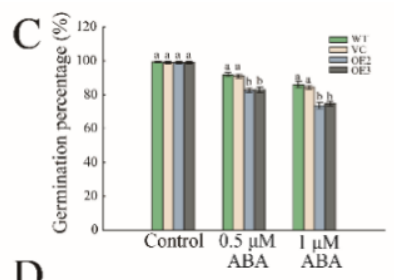

D

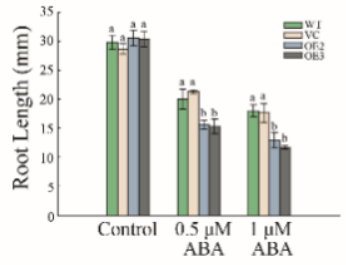

$\mathrm{E}$

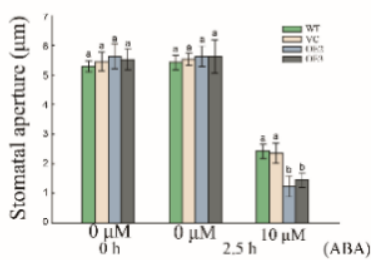

OE2
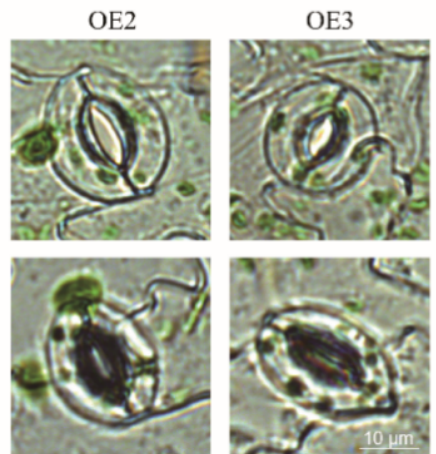

Figure 6. The stomatal aperture of PwNAC11 overexpression lines was positively modulated by ABA. (A) Germination assays for different lines under different concentrations of ABA treatments. (B) Root length assays for different lines under different concentrations of ABA treatments. (C) Quantification of germination percentage under ABA treatments. (D) Quantification of root length under ABA treatments. (E) Quantitative comparisons of stomatal aperture calculated by ImageJ software. (F) The observation of stomatal aperture of OE, WT and VC lines photographed by biomicroscope (DM2500, Leica). Different letters indicate the significant differences at $p<0.05$.

Recent studies indicated that increased ABA content could promote stomatal closure to improve the drought tolerance of plants [31]. We further observed and calculated the level of close stomata by exogenously applying ABA in different lines. The results showed that there was no distinct discrepancy under normal light conditions. Once adding $10 \mu \mathrm{M}$ $\mathrm{ABA}$, however, an acceleration of stomatal closure occurred in all plants, of which the $\mathrm{OE}$ 
lines exhibited a more obvious response to exogenous $\mathrm{ABA}$ treatment (Figure 6E,F). These results indicated that $P w N A C 11$ overexpression lines were more sensitive to exogenous ABA.

\subsection{PwNAC11 Interacts with ABF3 and DREB2A}

According to the prediction of the STRING and related reports of PwNAC11 homologous genes in Arabidopsis, five proteins were selected for potential interaction verification (Figure 7A). The transcriptional activity results showed that only pGBKT7-ANAC019 could grow well on selective medium and activate the reporter gene expression, which implied that ANAC019 has transcriptional activity, but not for other chosen proteins (Figure S2). Subsequent yeast two-hybrid $(\mathrm{Y} 2 \mathrm{H})$ assays revealed that the yeast cells containing pGADT7PwNAC11 + pGBKT7-ABF3 and pGADT7-PwNAC11 + pGBKT7-DREB2A grew well on $\mathrm{SD} /$ Trp-Leu-His-Ura selective medium (Figure 7B), suggesting that PwNAC11 can interact with ABF3 and DREB2A in yeast. Bimolecular fluorescence complementation (BiFC) assay further demonstrated that the YFP signals were observed in the nuclei of tobacco leaves coexpressing PwNAC11 and ABF3 or DREB2A (Figure 7C), which confirmed that PwNAC11 physically interacted with ABF3 or DREB2A in vivo.

A

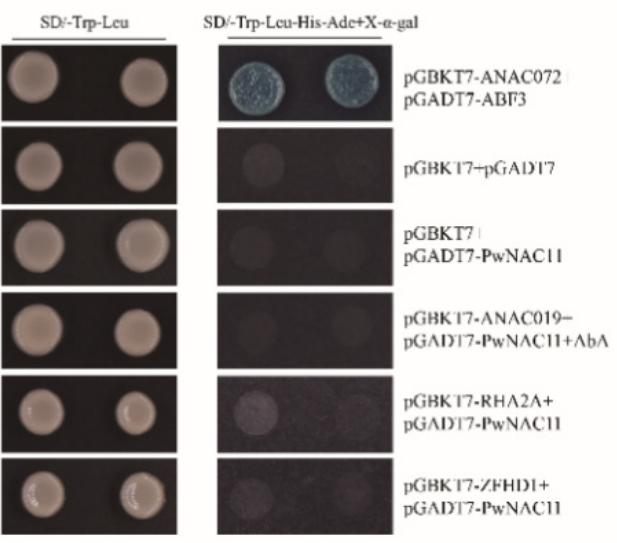

B
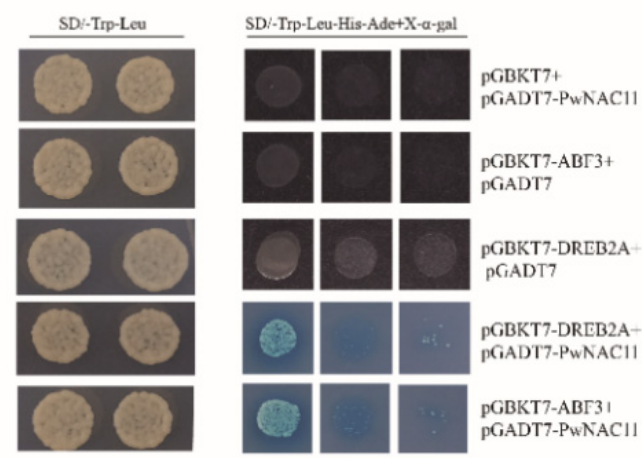
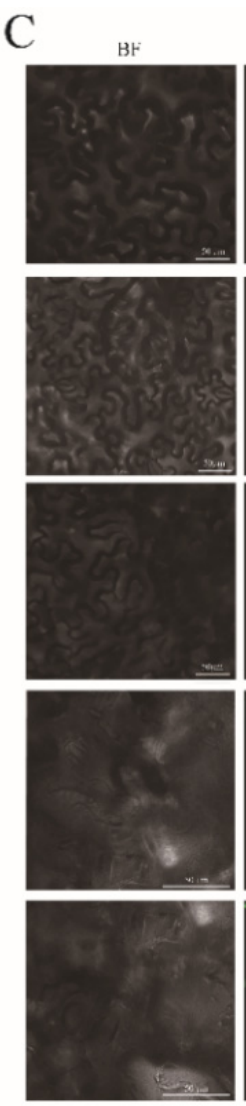
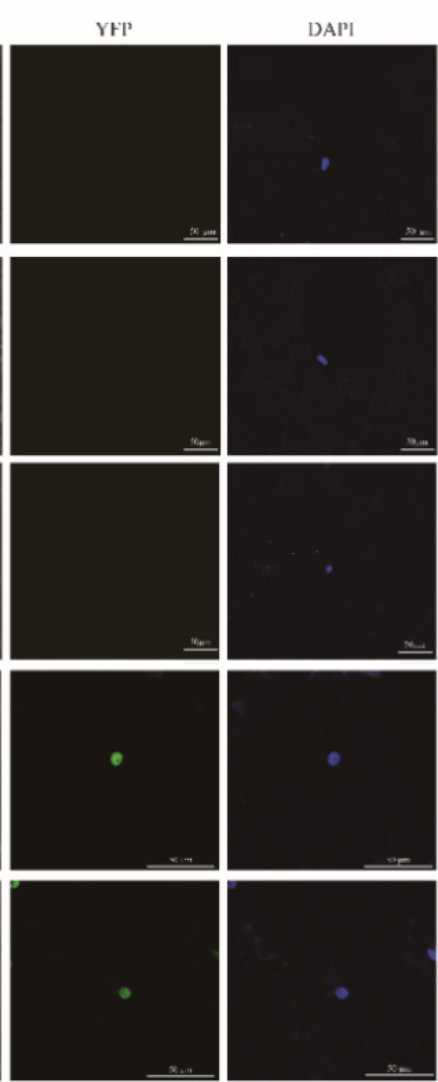
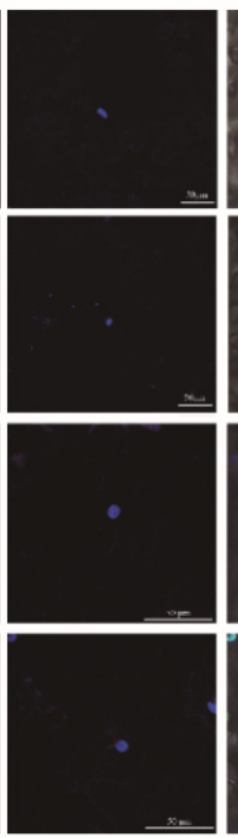
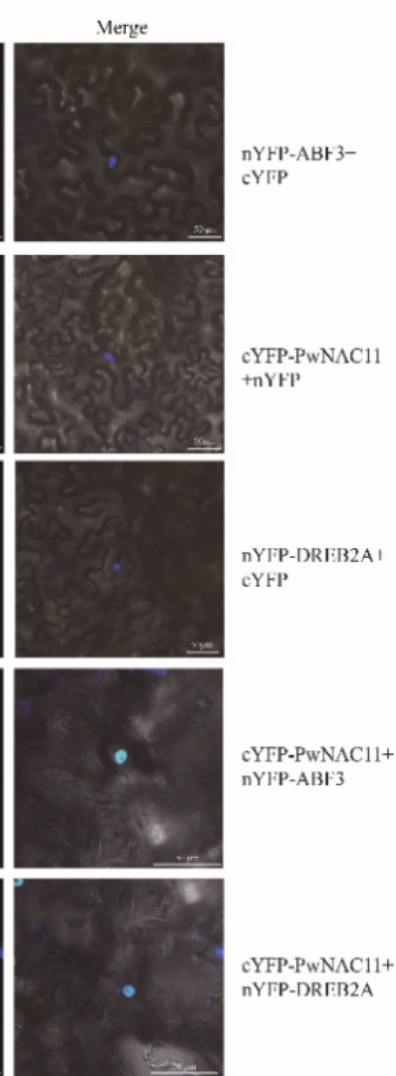

Figure 7. PwNAC11 interacts with ABF3 and DREB2A. (A) Yeast two-hybrid assays for PwNAC11 with ANAC019, RHA2A and ZFHD1. The yeast strains containing pGADT7-ABF3 and pGBKT7-ANAC072 fusion plasmids were used as positive control. Empty pGADT7 and pGBKT7 were used as negative control. (B) Yeast two-hybrid assays for PwNAC11 with ABF3 and DREB2A. (C) BiFC assays for PwNAC11 and ABF3/DREB2A interactions. Bars represent $50 \mu \mathrm{m}$. The C-terminal or $\mathrm{N}$-terminal of YFP was used as negative control.

\subsection{PwNAC11 and ABF3 Combine with the Promoter Region of ERD1}

Considering that ERD1 could be activated by ANAC019/ANAC072 in Arabidopsis [32], and the expression of ERD1 was prominently upregulated in PwNAC11 OE lines, we subsequently conducted the yeast one-hybrid ( $\mathrm{Y} 1 \mathrm{H})$ assays to confirm the regulation of 
PwNAC11 on ERD1 promoter region. In contrast with the negative control, both pGADT7PwNAC11 + pAbAi-ERD1pro and pGADT7-ABF3 + pAbAi-ERD1pro yeast strains could grow on SD/Ura-Leu + AbA selective medium (Figure S3), suggesting both PwNAC11 and ABF3 can combine with the ERD1 promoter (Figure 8A,B). As ABRE and DRE motifs were enriched in the promoter region of $E R D 1$, we performed another $\mathrm{Y} 1 \mathrm{H}$ assay to test whether PwNAC11 and ABF3 could bind to the specific cis-elements of ERD1 promoter. The results showed that both PwNAC11 and ABF3 could specifically combine with ABRE motifs, but neither PwNAC11 nor ABF3 could bind to the DRE motifs (Figure 8C). These results implied that $\mathrm{PwNAC11}$ and $\mathrm{ABF} 3$ synergistically regulate the ERD1 expression by binding to the ABRE motifs. Furthermore, we performed dual-luciferase assays in transformed tobacco. As is shown in Figure 8D, the expression of PwNAC11 promoted the activation of reporter gene by 2.67-fold than empty vector in tobacco, while ABF3 limitedly activated the LUC expression. The simultaneous expression of PwNAC11 and ABF3 together enhanced the activation of ERD1 promoter than PwNAC11 alone, which were 3.04 times higher than in the control group (Figure $8 \mathrm{D}$ ). These results proved that PwNAC11 functions as a positive regulator of the expression of ERD1, and co-expression of PwNAC11 and ABF3 further improves this activation by binding to the ABRE motifs. Moreover, co-expression of PwNAC11 and DREB2A also activated the expression of ERD1 promoter (Figure $8 \mathrm{E}$ ), suggesting that the regulation of PwNAC11 on ERD1 was also involved in an ABA-independent signaling pathway.

A

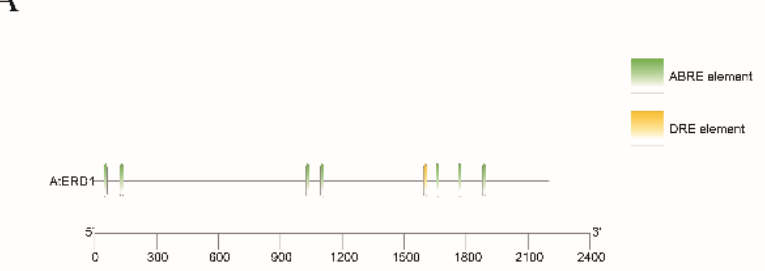

B

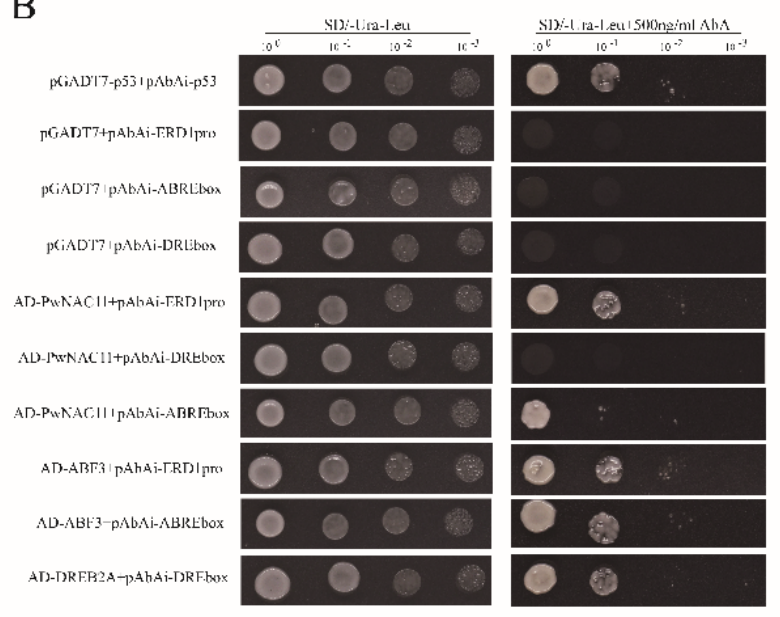

$\mathrm{C}$
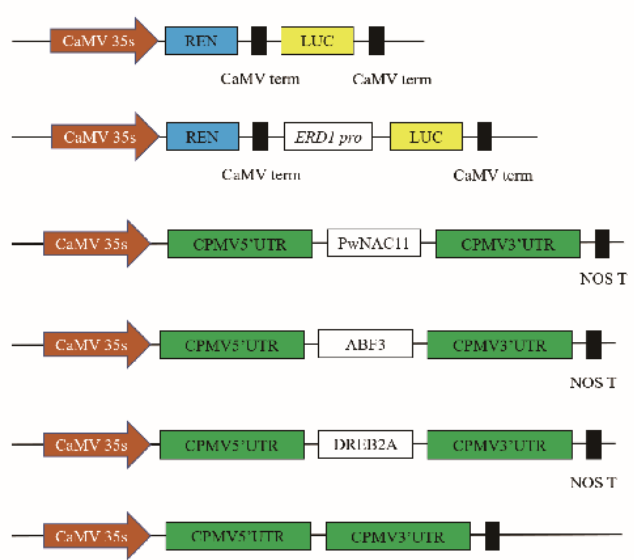

D

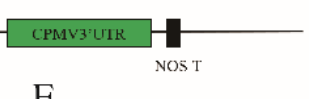

$\mathrm{E}$

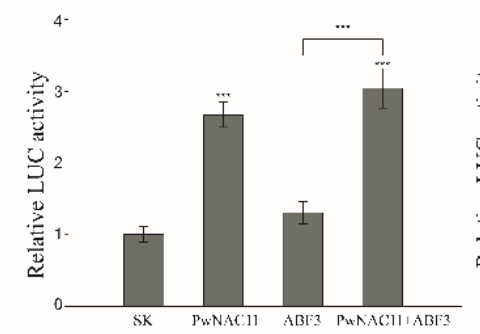

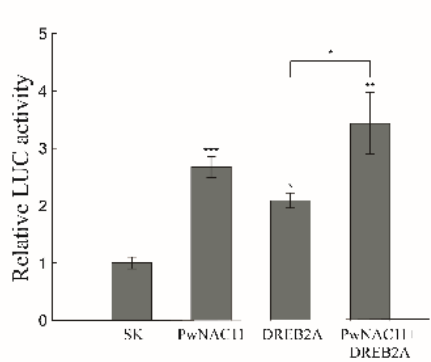

Figure 8. PwNAC11 and ABF3 cooperatively activates ERD1 transcription. (A) Diagram of the ERD1 promoter. The seven ABREs detected in ERD1 promoter are indicated in green and one DRE is marked in yellow. (B) Yeast one-hybrid assays for the interaction of PwNAC11 with ERD1 promoter, ABRE and DRE element. Aureobasidin A (AbA) of 500ng/mL was used to inhibit autoactivation. pGADT7-p53 and pAbAi-p53 were used as positive control. Empty PGADT7 was used as negative control. (C) Sketch map of the double-effector and reporter plasmids in dual-luciferase reporter assay. (D) Dual-luciferase assays for PwNAC11 and ABF3 interactions. (E) Dual-luciferase assays for PwNAC11 and DREB2A. The data are represented as means \pm standard error from three independent replications. The grey lines indicate the comparisons and asterisks indicate significant differences compared with SK $\left({ }^{*} p<0.05 ;{ }^{* *} p<0.01\right.$; $\left.{ }^{* * *} p<0.001\right)$. 


\subsection{PwNAC11 Overexpression Delays Flowering}

To test whether overexpression of PwNAC11 affected the growth and development of plants, some relative parameters were calculated. Under nonstress conditions, the rosette leaf morphology, plant height and pod length of different lines showed almost no differences (Figure S4A,B,D,E). However, the OE lines showed a 4-5-day delayed flowering phenotype compared with the WT and VC groups (Figure S4C). Meanwhile, we also examined the expression of some flowering-related genes by qRT-PCR and found that $L F Y, F Y$ and AP1 were significantly downregulated in 20-day-old transgenic Arabidopsis (Figure S4G-I). These results indicated that the delayed flowering phenotype was associated with the suppressed expression of flowering-related genes in PwNAC11 overexpression lines.

\section{Discussion}

\subsection{PwNAC11 Positively Regulates Drought Resistance in Transgenic Arabidopsis}

For most woody plants, it is still a great challenge to conducting functional verification in a homologous way via establishing transformation platforms [29]. Alternatively, we can still explore the gene function by heterogeneously transforming model plants. NAC transcription factors are reported to be involved in abiotic stress responses in various plant species, including model plants, crops and woody plants. Our previous work revealed that $P w N A C 2$, cloned from $P$. wilsonii, could interact with PwRFCP1 to positively regulate abiotic stress response and enhance drought and salt resistance in transgenic Arabidopsis [28]. Another NAC, TF PwNAC30, acted as a negative regulator in plant responses to abiotic stress. Overexpression of PwNAC30 apparently reduced the survival rates of transgenic Arabidopsis when subjected to drought and salt treatment [29], suggesting the distinct role and multiple function of different members of the NAC family from $P$. wilsonii in response to abiotic stress. Here, based on the high-throughput RNA-seq of the expression profiles of P. wilsonii in the absence or presence of drought treatments [30], we identified PwNAC11 as one of the most differentially expressed genes (DEGs) on a transcriptional level, which is a homologue of Arabidopsis NAC transcription factors ANAC019 and ANAC072 (RD26). Protein multiple sequence alignment and BLAST analysis showed that the conserved $\mathrm{N}$-terminal of PwNAC11 protein had a typical NAM domain and its C-terminal was comparatively variable. Both the full length and C-terminal regions of PwNAC11 have transcriptional activation activity (Figure 1). Subcellular localization demonstrated that PwNAC11 was a nuclear-located transcriptional activator, which was consistent with most NAC transcription factors [33]. The transcript level of PwNAC11 displayed a great increase at $3 \mathrm{~h}$ when $P$. wilsonii seedlings were exposed to drought stress, indicating its potential role in early response to abiotic stress. Furthermore, overexpression of PwNAC11 in Arabidopsis considerably improved drought stress tolerance both for seedlings and adult plants. Under PEG treatment, the growth of the tested seedlings was inhibited to some extent, but the $\mathrm{OE}$ lines showed significantly higher germination rates, stronger vitality and longer root length compared with the WT and VC lines. Moreover, the survival rates of the OE lines were more than $60 \%$ higher than the WT and VC groups at the adult stage after dehydration followed by re-watering. Additionally, the RWC of the adult OE plants was nearly $20 \%$ higher than the control group (Figure 3). These results demonstrated that PwNAC11 functions as a positive regulator in response to drought stress throughout plants' growth and development stages.

It is well known that drought can cause the accumulation of large amounts of ROS (mainly $\mathrm{H}_{2} \mathrm{O}_{2}$ and $\mathrm{O}_{2}^{-}$) in chloroplasts and mitochondria [34]. The excessive accumulation of ROS can cause serious peroxidation damage to plant cell membranes. Therefore, ROS eliminating efficiency is an essential indicator of plant resistance to drought stress. In our study, the NBT and DAB staining for $\mathrm{H}_{2} \mathrm{O}_{2}$ and $\mathrm{O}_{2}{ }^{-}$assays proved that ROS was slightly accumulated in the OE lines but accumulated extensively in WT and VC lines (Figure 4). Further measurement of enzymatic activities indicated that the activity of CAT, POD and SOD were upregulated in the OE lines compared to the lesser accumulation 
of MDA. These results indicated that PwNAC11 inhibits membrane lipid peroxidation via activating the ROS-scavenging system and decreasing the accumulation of ROS in resistance to drought stress.

Chlorophyll fluorescence is another important parameter reflecting the function of Photosystem II (PSII) and the transfer efficiency of electrons from PSII to PSI [35]. The $\mathrm{Fv} / \mathrm{Fm}$ ratio and $\Phi P S I I$ value represent the potential maximum light energy conversion efficiency and actual quantum yield of PSII, respectively [36,37]. However, the delicate photosynthetic system is frequently affected by the external environment, especially drought stress, which can decrease the Fv/Fm ratio and $\Phi P S I I$ value. In our study, despite these indicators of different samples showing a declining trend under drought conditions, the PwNAC11 OE lines displayed higher Fv/Fm, ФPSII and chlorophyll content compared with the WT and VC groups. Additionally, significantly, the values of $\Phi P S I I$ in transgenic lines remained almost unchanged whether in the presence or absence of drought treatment, suggesting the minimizing damage to the photosynthesis apparatus of PSII in PwNAC11 OE lines and PwNAC11 can stabilize photosynthetic energy utilization under drought conditions. Meanwhile, the NPQ, an indicator of plant to disperse excess light energy in the form of heat to protect itself under adverse conditions, showed no significant differences under normal and dehydration conditions in control groups, while PwNAC11 OE lines showed increased NPQ values after drought treatment. These results verified the complete network of photoprotective mechanism in OE lines [38]. Recent studies revealed that the inhibition of light energy conversion could produce more excess excitation energy and promote the accumulation of ROS in plant [39]. In grape, Sun et al. found that the inhibition of Alternative oxidase (AOX) pathway increased the accumulation of $\mathrm{H}_{2} \mathrm{O}_{2}$, thus resulting in the photoinhibition under heat conditions [40]. Overexpression of Arabidopsis AtCBF3 enabled transgenic potatoes to possess a favorable ROS removing ability, which decreased the degree of photoinhibition and enhanced heat resistance [41]. Therefore, we conclude that the efficient ROS-scavenging system in PwNAC11 OE lines may partly contribute to the stable light energy conversion efficiency under drought conditions.

Many NAC TFs involved in stress response were reported to have an effect on plant development or flowering. For example, EsNAC1, an NAC TF isolated from E. salsugineum, was proven as a positive regulator of salt and oxidative stress but inhibited the process of vegetative growth [42]. Additionally, MLNAC5, a stress-related transcription factor from Miscanthus, improved drought and cold tolerance in transgenic Arabidopsis but inhibited the growth of the plant and accelerated the process of leaf senescence [43]. The overexpression of sugarcane NAC transcription factor ScNAC23 in Arabidopsis accelerated flowering compared with WT plants [44]. Our previous study also revealed that another P. wilsonii transcription factor, PwNAC2, enhances drought and salt tolerance in plants and can interact with PwRFCP1 to co-regulate flowering via regulating the expression of SOC1, FLC and FT [28]. These results indicate that the different NAC transcription factors function differentially, and some of them can balance and coordinate the regulation of plant growth and flowering and coping with adverse stress. In this study, a late flowering phenotype was discovered in 35S: PwNAC11 OE lines. Further investigation demonstrated that PwNAC11 delayed flowering time by inhibiting the expression of genes such as $L Y F$, FT and AP1. Interestingly, the growth retardation disappeared when the plants were subjected to drought conditions (Figure 3A). Previous research showed that under nonstress conditions the growth inhibition phenotype was partly affected by the constitutive expression of $35 S$ promoter [45]. Under normal conditions, for example, ectopic expression of 35S: AtDREB1A in Salvia miltiorrhiza inhibited plant growth but the overexpression of stress-inducible RD29A: AtDREB1A caused no growth suppression. Therefore, we cannot exclude the effect of $35 \mathrm{~S}$ promoter on flowering time in PwNAC11 transgenic Arabidopsis. To further determine whether the stunted growth phenotype was mainly caused by functional PwNAC11 expression or $35 \mathrm{~S}$ promoter, knock-out studies are required to verify this hypothesis and decipher the role of PwNAC11 on flowering regulation in P. wilsonii in the future. In addition, considering most transcription factors are simultaneously involved 
in plant growth, development and response to stress, the stress-induced promoters or self-gene promoters are probably recommended for plant gene transformation to reduce the impact on the development process of the plant itself.

\subsection{PwNAC11 Improves Drought Tolerance in an ABA-Dependent Manner}

Plants' responses to drought stress are usually controlled by hormonal signals, in which $\mathrm{ABA}$ acts as a mediator to regulate a range of processes in stress-signaling pathways [46]. CmBBX19, a zinc finger protein, suppressed drought resistance in chrysanthemum in an ABA-dependent way [47]. At a translational level, alternative splicing of rice NAC transcription factor ONAC054 led to premature termination of translation in response to exogenous $\mathrm{ABA}$, thus producing more $\mathrm{ABA}$-responsive ONAC054 $\beta$ variants to positively modulate the homeostasis [48]. In our study, expression of PwNAC11 was greatly upregulated especially at $3 \mathrm{~h}$ after ABA treatment. PwNAC11 overexpression lines conferred hypersensitivity to ABA at an earlier phase than the WT and VC groups. Meanwhile, some ABA synthesis genes, such as NCED3 and ABI5, showed transcriptional abundance after exposure to PEG. These results implied that PwNAC11 is likely to participate in water-deficit regulation in an ABA-dependent pathway. In addition, previous investigations revealed that most TFs were involved in the regulation of stomata closure under the control of ABA signals. The heterologous expression of AINAC1 in Arabidopsis increased the endogenous ABA level, thus resulting in an enhanced percentage of close stomata and reduced water loss [49]. CsMYB61, a MYB TF from Citrus sinensis, was implicated in the process of stomatal closure. Overexpression of CsMYB61 driven by a stomata-specific promoter conferred a repressed opening of stomata pores, especially under the treatment of exogenous ABA [50]. Similarly, AtMYB61 transgenic Arabidopsis displayed a higher percentage of closed stomata under drought treatment [51]. Here, we found that PwNAC11 acted in a similar way to regulate ABA-induced stomatal closure. Overexpression of PwNAC11 transcript in Arabidopsis led to a significant increase in the number of close stomata (Figure 6). It was reported that stomatal closure is generally triggered by drought-induced hormone ABA as well as the application of exogenous ABA [52,53]. Our results also demonstrate that PwNAC11 functions as an ABA signal factor by promoting stomatal closure and ABA synthesis to directly or indirectly improve plants' tolerance to drought stress.

The ABF TFs are considered as pivotal recipients of ABA signaling and function downstream of various ABA-mediated stress response, especially drought and salt stresses $[54,55]$. ABI5, an important ABA synthesis gene, is governed by AtABF3 in Arabidopsis, which could enhance salt stress tolerance by activating the ABA signal pathway [56]. In Populus euphratica, PeABF3 could activate the expression of PeADF5 to enhance drought tolerance and promote ABA-induced stomatal closure [57]. Although different ABFs exhibited high levels of homology, they could also work antagonistically in definite conditions to generate a negative feedback effect. Pear ABF protein PpyABF3 can activate PpyDAM3 expression by binding to the ABRE motif on PpyDAM3 promoter, while the binding affinity of PpyABF3 to PpyDAM3 promoter was disturbed by interacting with PpyABF2, thus revealing the antagonistic regulatory network of $\mathrm{ABFs}$ towards downstream genes [58]. In the present study, we found that PwNAC11 could physically interact with AtABF3 by $\mathrm{Y} 2 \mathrm{H}$ and $\mathrm{BiFC}$ assay, which is consistent with another homologous gene, ANAC072, in Arabidopsis. ANAC072 participated in the ABA-responsive signaling pathway by interacting with ABF3 [59]. The data showed the conservation of function and further proves that NAC TFs could perform similar functions in both woody plants and Arabidopsis. However, ANAC072 was reported to promote leaf senescence and accelerate chlorophyll degradation, which was not observed in PwNAC11 transgenic lines.

\subsection{The Regulatory Networks of ABA-Induced Drought Resistance Mediated by PwNAC11}

TF acts as a transcriptional activator/repressor by specifically binding to cis-acting elements in the promoters of target genes. Accordingly, it will enable us to uncover 
the unknown regulatory mechanisms by identifying the relative target genes. ERD1 encoded a homologous protein of ATP binding subunit in Escherichia coli [13], and the expression of ERD1 was much upregulated by abiotic stress or dark-induced senescence. In Arabidopsis, a zinc finger homeodomain TF (ZFHD1) could enhance the expression of $E R D 1$ by binding to its promoter region, which contributed to an obvious improvement of drought stress endurance in the ZFHD1 overexpression lines [60]. Likewise, we found that PwNAC11 bound to the promoter of ERD1 and activated ERD1 expression. RT-qPCR analysis also showed that the expression of PwNAC11 reached its peak at $3 \mathrm{~h}$ after PEG treatment, while at $6 \mathrm{~h}$ for ERD1. We next performed a Y1H assay to prove that PwNAC11 could specifically bind to ABRE motifs instead of DRE motifs. These results provide genetic evidence that PwNAC11 functions as a transcriptional activator upstream of ERD1, leading to enhanced dehydration resistance in an ABA-dependent manner. In addition, our results also confirmed the interaction between PwNAC11 and ABF3 (Figure 7), both of which could bind to the ABRE elements (Figure 8), but whether PwNAC11 and ABF3 simultaneously bind to the same ABRE element on ERD1 promoter, and whether the interaction of PwNAC11 and ABF3 interferes with the binding affinity to target genes remains to be further investigated. Nevertheless, the expression of PwNAC11 promoted the activation of the reporter gene by 2.67 -fold greater than empty vector in tobacco, while ABF3 limitedly activated LUC expression. However, the simultaneous expression of PwNAC11 and ABF3 together enhanced the activation of the ERD1 promoter more than PwNAC11 alone (Figure 8D). These results showed that PwNAC11 positively regulates plant response to early drought stress and the interaction of PwNAC11 and ABF3 further improves this activation by binding to the ABRE motifs. Generally, ABF3 can interact physically with diverse components to promote or inhibit the expression of downstream targets, especially stress-responsive genes. For example, in Arabidopsis, AtSOC1 was ubiquitously expressed in different vegetative tissues and accelerated flowering. AtABF3 could form a complex with NF-Y TF and activated the expression of AtSOC1, and this phenotype was totally abolished in $n f-y c 3$ yc4 yc9 mutants [61]. Similarly, the physical interaction between CmBBX19 and CmABF3 in Chrysanthemum withheld the activation of $C m R A B 18$, resulting in decreased tolerance to drought stress [47].

However, our results by no means suggest the minimal role of $A B A$-independent pathway present in PwNAC11-mediated drought stress response, since DRE-BINDING PROTEIN 2A (DREB2A) also physically interacted with PwNAC11 by $\mathrm{Y} 2 \mathrm{H}$ and BiFC assay in this study (Figure 7). It was noteworthy that dehydration-responsive element (DRE) also exists in the promoter of $E R D 1$, which is recognized as an essential cis-element for ABA-independent regulation in response to dehydration and heat stresses [62]. In woody plants, the expression of BpDREB2, a transcription factor of DREB protein family in Broussonetia papyrifera, was remarkably increased under salt and dehydration conditions, but no obvious change was observed under ABA treatment. Importantly, overexpression of $B p D R E B 2$ in Arabidopsis improved its salt and freezing tolerance [63]. Similar results were found in Jatropha curcas. The expression patterns of JcDREB showed that it was induced by cold, drought and salt stresses, not by ABA signal [64]. However, some reports have also suggested that DREB transcription factors can be mediated in both ABA-dependent and ABA-independent pathways in response to abiotic stress. In Leymus chinensis, the transcript of $L c D R E B 3 a$ accumulated in response to ABA treatments and the protein was shown to bind to DRE motifs, indicating that $L C D R E B 3 a$ was involved in both ABA-dependent and ABA-independent signal transduction in the process of abiotic stress response. Here, we also found that PwNAC11 remarkably improved the transcription activity of ERD1 promoter by interacting with DREB2A rather than directly binding to the DRE motif. We thus speculate that the drought response of ERD1 is also modulated in an indirect ABAindependent approach, in which PwNAC11 may also participate. This assertion is also supported by the apparent up-regulation of DREB2A in transgenic Arabidopsis, whose expression levels are less dependent on ABA signals $[65,66]$. 
In addition, combined with the data in our study that PwNAC11 was dramatically induced at $3 \mathrm{~h}$ after PEG treatment, while ABF3 highly expressed at $12 \mathrm{~h}$ and DREB2A at $6 \mathrm{~h}$, we speculated that PwNAC11 plays a dominant role in positively regulating plants' responses to early drought stress, and ABF3 or DREB2A were subsequently induced and synergistically regulate the ERD1 expression. Based on these results and the function of homologous genes, we sketched the regulatory mechanism and linkage of ABA signals and the drought response pathway mediated by PwNAC11 (Figure 9). First, PwNAC11 functions to improve the expression of ABF3 or DREB2A at transcriptional-level control under drought stress, then the protein interaction between PwNAC11 and ABF3 strengthens the transcription of target genes by activating ABRE-containing promoters or indirectly activating DRE-containing promoters by interaction with DREB2A. The existence of such synergistic pathways could help plants to respond to environmental stress efficiently and rapidly, but it is necessary to further explore how precise networks PwNAC11 participate or contribute to the drought stress response via ABA-dependent or -independent pathways, which will provide further information on the drought tolerance mechanism of Picea wilsonii.
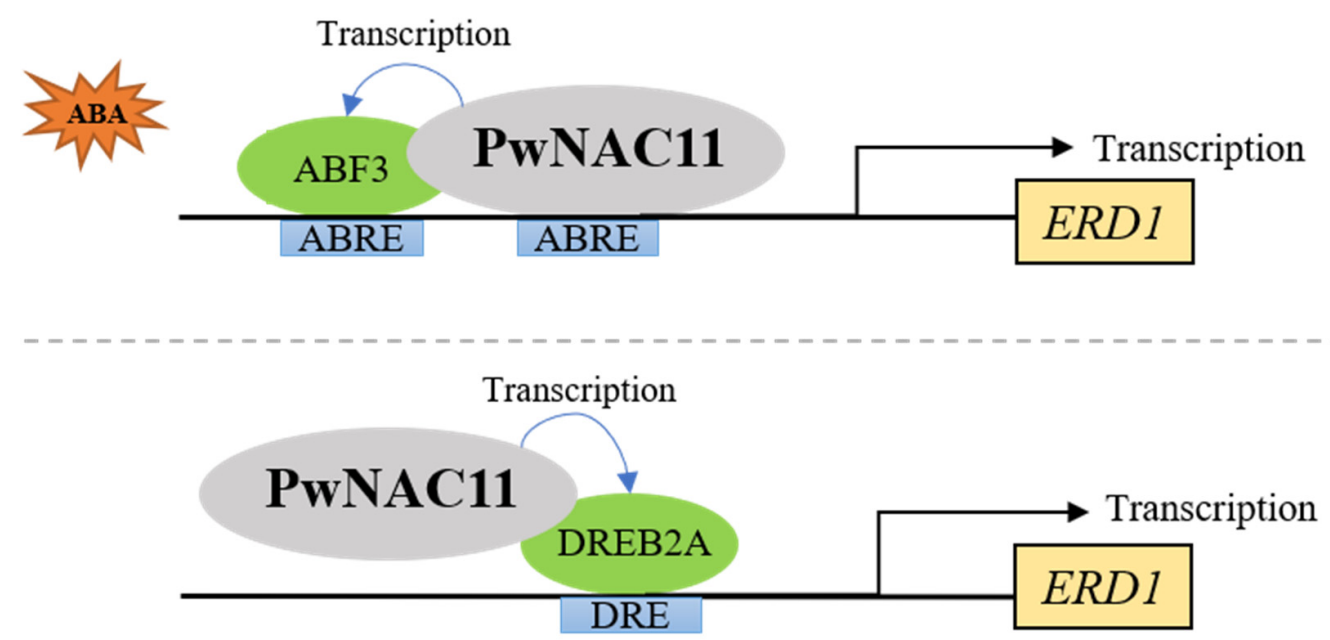

Figure 9. Proposed model of ABA-dependent and ABA-independent abiotic stress responses via PwNAC11.

\section{Materials and Methods}

\subsection{Plant Materials and Gene Expression Analysis}

For RT-qPCR assay, the 4-week-old P. wilsonii seedlings grown on nutrient soil were watered with $20 \%$ polyethylene glycol (PEG) or treated with $100 \mu \mathrm{M}$ ABA solution and the 2-week-old Arabidopsis seedlings were watered with 10\% PEG solution. The whole plants were then harvested at $0,3,6$ and $12 \mathrm{~h}$ after treatment for RNA extraction. Total RNA was extracted from P. wilsonii or Arabidopsis with plant RNA extraction kit (Kangwei Century Biotechnology Co., Ltd., Beijing China) and first strand cDNA synthesis kit (ABM, Beijing, China), followed by RT-qPCR analysis. Step One plus Real-time PCR system (ABI, Vernon, CA, USA) was used to perform RT-qPCR reactions with SYBR Green Master Mix enzymes (ABI, Vernon, CA, USA). The $2-\Delta \Delta C T$ method was employed to calculate expression levels and all experiments were repeated 3 times for biological replications and 3 times for technical replications. PwEF-1 $\alpha$ in P. wilsonii and ACTIN in Arabidopsis were selected as housekeeping genes in qRT-PCR assay, respectively.

Before sowing in the MS medium, all seeds were sterilized by $5 \% \mathrm{NaClO}$ solution for $20 \mathrm{~min}$. After 3 days dark treatment, the seeds were then placed in a $21^{\circ} \mathrm{C}$ incubator under a 16-h light/8-h dark photoperiod at 70\% relative humidity. At 14-days-old, plants were transferred into soil. 


\subsection{Bioinformatic Analysis}

The sequences of PwNAC11 homologous proteins were obtained from NCBI database (https: / / www.ncbi.nlm.nih.gov / (accessed on 1st November 2018)). Multiple sequence alignments of $12 \mathrm{NAC}$ amino acid sequences were analyzed using Clustal X 1.83. The neighbor-joining method was subsequently used to construct the phylogenetic tree via MEGA-X (Mega Limited, Auckland, New Zealand) program.

\subsection{Plant Phenotype Experiments under Drought and ABA Treatments}

Seeds of Arabidopsis have Col-0 genetic background. To generate transgenic plants, full length of PwNAC11 CDS was cloned into pCAMBIA1205 vector, which was driven by 35S promotor [28]. The agrobacterium stains GV3101 containing PwNAC11-pCAMBIA1205 were subsequently transformed into Arabidopsis using floral dipping. Stable homozygous lines were selected by hygromycin resistance and all T3 seedings could survive on selective medium, in which two dependent lines named OE-2 and OE-3, with relatively higher expression levels, were chosen as experimental samples.

The WT and VC lines were set as controls and OE-2 and OE-3 lines were chosen for phenotype analysis. For the seed germination experiment, the disinfected seeds were sown on MS solid medium (100 seeds were sown for each line) containing various concentration of $\mathrm{ABA}(0,0.5,1.0 \mu \mathrm{M})$ and mannitol $(0,100,200,300 \mathrm{mM})$, respectively. After placing in a refrigerator at $4{ }^{\circ} \mathrm{C}$ for 3 days, the mediums were then transferred into an incubator as described above. The germination number was recorded once a day and the germination criterion was the exposure of radicle. For the root length experiment, the seeds of each line were sown in MS medium for germination and then transferred to MS medium supplemented with different concentrations of mannitol and ABA. For seedlings treated with drought stress, all of the lines were sown on MS medium for 2 weeks and then transferred to soil under normal conditions for another 14 days. For drought stress, the 4-week-old seedlings were dehydrated for 14 days and then re-watered for 3 days. Phenotypic differences were observed after 14 days of drought treatment and re-watering. Each experiment was repeated three times.

\subsection{Subcellular Localization Assay}

The CDS of PwNAC11 was constructed on pCAMBIA1205 vector, which was controlled by CaMV35S promotor. The RACK1A-RFP plasmid, a nuclear-located marker [29], and recombinant vector were transfected into Agrobacterium tumefaciens GV3101 cells, respectively, and then co-infiltrated into Nicotiana benthamiana leaves, which were then placed in the incubator at $25{ }^{\circ} \mathrm{C}$ (day) $/ 23{ }^{\circ} \mathrm{C}$ (night) for $48-72 \mathrm{~h}$. The TCS SP8 fluorescence microscope (Leica, Wetzlar, Germany) was used to detect GFP fluorescence. Three infiltrated leaves from two $N$. benthamiana were regarded as three biological repeats for each experiment.

\subsection{Trans-Acting Activity and YEAST Two-Hybrid (Y2H) Assay}

The complete PwNAC11 CDS and truncated N-terminal (pGBKT7-PwNAC11-1-153) or C-terminal (pGBKT7-PwNAC11-171-318) sequences of PwNAC31 were cloned into pGBKT7 vector, respectively. The assay was performed as previously described with modification [29]. Briefly, the plasmids were separately transformed into yeast AH109 cells with empty pGBKT7 vector as negative control. The yeast strains were firstly painted on $\mathrm{SD} /$-Trp medium and then transformed into SD/-Trp-His-Ade+X- $\alpha$-gal selective medium.

For $\mathrm{Y} 2 \mathrm{H}$ assay, similar transcriptional activity assays were conducted. The CDS of $A B F 3, Z F H D 1, R H A 2 A$ or $A N A C 019$ were cloned to $\mathrm{pGBKT7}$ vector as the prey and recombinant vectors were then selected on $\mathrm{SD} /$-Trp-His-Ade $+X$ - $\alpha$-gal medium. Full-length CDS of PwNAC11 was correspondingly constructed on pGADT7 vector as the bait. The recombinant $\mathrm{AD}$ and $\mathrm{BD}$ vectors were simultaneously inserted into yeast $\mathrm{AH} 109$ cells and selected on SD/-Trp-Ura-His-Ade+X- $\alpha$-gal medium. 


\subsection{Yeast One-Hybrid (Y1H) Assay}

Y1H assay was accomplished with the Matchmaker Gold Yeast One-Hybrid System Kit (TaKaRa, Beijing, China). Briefly, about $2 \mathrm{~kb} E R D 1$ promoter region and short tandem repeats containing core ABRE (ACGTG) and DRE (GTCGGC) motifs were constructed on pAbAi vector. The pGADT7-PwNAC11 or pGADT7-ABF3 vectors were transformed into competent cells of yeast containing the $E R D 1$ pro sequences. The recombinant vectors were selected on SD/-Ura-Leu+ AbA medium.

\subsection{Dual-Luciferase Assay}

Dual-luciferase assay was performed as previously described [67]. Briefly, the ERD1 promoter was cloned into pGreenII 0800-LUC and the CDS of PwNAC11, ABF3 or DREB2A was cloned into pGreenII 62-SK vector. The transfected Nicotiana benthamiana leaves were detected by Dual Luciferase Reporter Gene Assay Kit (Beyotime, Shanghai, China). The firefly luciferase (fLUC) and renilla luciferase (rLUC) were detected by Multiscan Spectrum (Inginite M Plex, TECAN). The empty pGreenII 0800-LUC vector was used as control and each experiment was repeated three times.

\subsection{Bimolecular Fluorescence Complementation Assay (BiFC)}

The complete CDS of PwNAC11 was cloned into PSPYCE vector to generate PwNAC11cYFP fusion protein and coding region of $A B F 3$ was similarly cloned into PSPYNE vector. The recombinant vectors were transformed into agrobacterium strains GV3101 and then co-infiltrated into Nicotiana benthamiana leaves as mentioned above. The GFP signal was observed through Leica TCS SP8 fluorescence microscope.

\subsection{Physiological Measurement}

The Diaminobenzidine (DAB) and Nitro Blue Tetrazolium (NBT) staining assays were conducted as previously described [68]. NBT Chromogen and Metal Enhanced DAB Substrate Kit were employed in this experiment (Solarbio, Beijing, China). Briefly, the leaves of different lines were immersed in the staining solution for $2 \mathrm{~h}$ in darkness. Then, the staining solution was replaced by $95 \%$ alcohol and the leaves were boiled until decolorization. The catalase, antioxidant enzymes' activities and proline content were measured by CAT, SOD, POD and Pro detection kits (Jiancheng Bioengineering Institute, Nanjing, China). The chlorophyll content was quantified by spectrometer as previously described [69]. The chlorophyll and fluorescence parameters of Arabidopsis leaves under drought stress were measured by handy portable fluorometer (PAM-2500, WALZ, Germany), and the fluorescence parameters were calculated as previously described [35]. Some parameters were calculated as follows: $\mathrm{NPQ}=\mathrm{Fm} / \mathrm{Fm}^{\prime}-1, \mathrm{Fv} / \mathrm{Fm}=(\mathrm{Fm}-\mathrm{Fo}) / \mathrm{Fm}$, $\Phi P S I I=\left(\mathrm{Fm}^{\prime}-\mathrm{F}\right) / \mathrm{Fm}^{\prime}$, in which Fo is the minimum recorded fluorescence intensity in the dark-adapted states. Fm and Fm' are the maximal recorded fluorescence intensity in the dark and light-adapted states, respectively.

\subsection{Stomatal Aperture Measurements}

Stomatal apertures were measured as described previously [70]. Leaves of Arabidopsis were pre-incubated in MES-KCl buffer for $2.5 \mathrm{~h}$ under normal light conditions, followed by MES-KCl buffer treatment alone or with $10 \mu \mathrm{M}$ ABA for another $3 \mathrm{~h}$. Stomatal pores of OE, WT and VC lines were photographed by light microscope DM2500 (Leica, Wetzlar, Germany).

\subsection{Statistical Analysis}

SPSS software version 18.0 (SPSS Corp, Chicago, IL, USA) was used for statistical analysis. Duncan's multiple range test was performed for statistical difference analysis with $p<0.05$ designated as significant difference. 
Supplementary Materials: Supplementary Materials can be found at https:/ /www.mdpi.com/ article/10.3390/ijms22136952/s1.

Author Contributions: L.Z. conceived the project and L.Z. and M.Y. designed the experiments; J.L. collected the samples; M.Y., J.L., B.D., A.W. and M.Z. performed the experiments; M.Y. conducted the data analysis and drafted the manuscript; L.Z. revised the manuscript. All authors have read and agreed to the published version of the manuscript.

Funding: This research was funded by a grant from the Agricultural Ministry of China, grant number 2016ZX08009-003.

Institutional Review Board Statement: Not applicable.

Informed Consent Statement: Not applicable.

Data Availability Statement: No new data were created or analyzed in this study. Data sharing is not applicable to this article.

Acknowledgments: We thank Dapeng Zhang (College of Life Sciences, Tsinghua University) for providing the pGreenII 62-SK and pGreenII 0800-LUC vector.

Conflicts of Interest: The authors declare that they have no conflict of interest.

\section{References}

1. Guo, Y.Y.; Yu, H.Y.; Kong, D.S.; Yan, F.; Zhang, Y.J. Effects of drought stress on growth and chlorophyll fluorescence of Lycium ruthenicum Murr. seedlings. Photosynthetica 2016, 54, 524-531. [CrossRef]

2. Zhao, P.; Hou, S.; Guo, X.; Jia, J.; Yang, W.; Liu, Z.; Chen, S.; Li, X.; Qi, D.; Liu, G.; et al. A MYB-related transcription factor from sheepgrass, LcMYB2, promotes seed germination and root growth under drought stress. BMC Plant Biol. 2019, 19, 954. [CrossRef]

3. Du, H.; Huang, F.; Wu, N.; Li, X.; Hu, H.; Xiong, L. Integrative Regulation of Drought Escape through ABA-Dependent and -Independent Pathways in Rice. Mol. Plant 2018, 11, 584-597. [CrossRef] [PubMed]

4. Yu, Y.; Wang, L.; Chen, J.; Liu, Z.; Park, C.-M.; Xiang, F. WRKY71 Acts Antagonistically Against Salt-Delayed Flowering in Arabidopsis thaliana. Plant Cell Physiol. 2017, 59, 414-422. [CrossRef]

5. Blum, A. Effective use of water (EUW) and not water-use efficiency (WUE) is the target of crop yield improvement under drought stress. Field Crop. Res. 2009, 112, 119-123. [CrossRef]

6. Olsen, A.N.; Ernst, H.A.; Leggio, L.L.; Skriver, K. NAC transcription factors: Structurally distinct, functionally diverse. Trends Plant Sci. 2005, 10, 79-87. [CrossRef]

7. Puranik, S.; Sahu, P.P.; Srivastava, P.S.; Prasad, M. NAC proteins: Regulation and role in stress tolerance. Trends Plant Sci. 2012, 17, 369-381. [CrossRef] [PubMed]

8. Ooka, H.; Satoh, K.; Doi, K.; Nagata, T.; Otomo, Y.; Murakami, K.; Matsubara, K.; Osato, N.; Kawai, J.; Carninci, P.; et al. Comprehensive Analysis of NAC Family Genes in Oryza sativa and Arabidopsis thaliana. DNA Res. 2003, 10, 239-247. [CrossRef] [PubMed]

9. Raja, V.; Majeed, U.; Kang, H.; Andrabi, K.I.; John, R. Abiotic stress: Interplay between ROS, hormones and MAPKs. Environ. Exp. Bot. 2017, 137, 142-157. [CrossRef]

10. Li, S.; Gao, J.; Yao, L.; Ren, G.; Zhu, X.; Gao, S.; Qiu, K.; Zhou, X.; Kuai, B. The role of ANAC072 in the regulation of chlorophyll degradation during age- and dark-induced leaf senescence. Plant Cell Rep. 2016, 35, 1729-1741. [CrossRef] [PubMed]

11. Zhu, M.; Chen, G.; Zhou, S.; Tu, Y.; Wang, Y.; Dong, T.; Hu, Z. A New Tomato NAC (NAM/ATAF1/2/CUC2) Transcription Factor, SINAC4, Functions as a Positive Regulator of Fruit Ripening and Carotenoid Accumulation. Plant Cell Physiol. 2013, 55, 119-135. [CrossRef]

12. Mao, C.; Lu, S.; Lv, B.; Zhang, B.; Shen, J.; He, J.; Luo, L.; Xi, D.; Chen, X.; Ming, F. A Rice NAC Transcription Factor Promotes Leaf Senescence via ABA Biosynthesis. Plant Physiol. 2017, 174, 1747-1763. [CrossRef]

13. Tran, L.-S.P.; Nakashima, K.; Sakuma, Y.; Simpson, S.D.; Fujita, Y.; Maruyama, K.; Fujita, M.; Seki, M.; Shinozaki, K.; YamaguchiShinozaki, K. Isolation and Functional Analysis of Arabidopsis Stress-Inducible NAC Transcription Factors That Bind to a Drought-Responsive cis-Element in the early responsive to dehydration stress 1 Promoter. Plant Cell 2004, 16, $2481-2498$. [CrossRef]

14. Ma, J.; Wang, L.-Y.; Dai, J.-X.; Wang, Y.; Lin, D. The NAC-type transcription factor CaNAC46 regulates the salt and drought tolerance of transgenic Arabidopsis thaliana. BMC Plant Biol. 2021, 21, 11. [CrossRef] [PubMed]

15. Mao, H.; Li, S.; Wang, Z.; Cheng, X.; Li, F.; Mei, F.; Chen, N.; Kang, Z. Regulatory changes in TaSNAC8-6A are associated with drought tolerance in wheat seedlings. Plant Biotechnol. J. 2019, 18, 1078-1092. [CrossRef] [PubMed]

16. Thirumalaikumar, V.P.; Devkar, V.; Mehterov, N.; Ali, S.; Ozgur, R.; Turkan, I.; Mueller-Roeber, B.; Balazadeh, S. NAC transcription factor JUNGBRUNNEN1 enhances drought tolerance in tomato. Plant Biotechnol. J. 2017, 16, 354-366. [CrossRef] [PubMed]

17. Hu, P.; Zhang, K.; Yang, C. BpNAC012 Positively Regulates Abiotic Stress Responses and Secondary Wall Biosynthesis. Plant Physiol. 2019, 179, 700-717. [CrossRef] [PubMed] 
18. Ehong, Y.; Ezhang, H.; Ehuang, L.; Eli, D.; Esong, F. Overexpression of a Stress-Responsive NAC Transcription Factor Gene ONAC022 Improves Drought and Salt Tolerance in Rice. Front. Plant Sci. 2016, 7, 4. [CrossRef]

19. Xu, Z.-Y.; Kim, S.Y.; Hyeon, D.Y.; Kim, D.H.; Dong, T.; Park, Y.; Jin, J.B.; Joo, S.-H.; Hong, J.C.; Hwang, D.; et al. The Arabidopsis NAC Transcription Factor ANAC096 Cooperates with bZIP-Type Transcription Factors in Dehydration and Osmotic Stress Responses. Plant Cell 2013, 25, 4708-4724. [CrossRef]

20. Dodd, I.C.; Egea, G.; Watts, C.W.; Whalley, W.R.; Cegarra, G.E. Root water potential integrates discrete soil physical properties to influence ABA signalling during partial rootzone drying. J. Exp. Bot. 2010, 61, 3543-3551. [CrossRef]

21. Tuteja, N. Abscisic Acid and Abiotic Stress Signaling. Plant Signal. Behav. 2007, 2, 135-138. [CrossRef] [PubMed]

22. Xue-Xuan, X.; Hong-Bo, S.; Yuan-Yuan, M.; Gang, X.; Jun-Na, S.; Dong-Gang, G.; Cheng-Jiang, R. Biotechnological implications from abscisic acid (ABA) roles in cold stress and leaf senescence as an important signal for improving plant sustainable survival under abiotic-stressed conditions. Crit. Rev. Biotechnol. 2010, 30, 222-230. [CrossRef] [PubMed]

23. Sato, H.; Takasaki, H.; Takahashi, F.; Suzuki, T.; Iuchi, S.; Mitsuda, N.; Ohme-Takagi, M.; Ikeda, M.; Seo, M.; Yamaguchi-Shinozaki, K.; et al. Arabidopsis thaliana NGATHA1 transcription factor induces ABA biosynthesis by activating NCED3 gene during dehydration stress. Proc. Natl. Acad. Sci. USA 2018, 115, E11178-E11187. [CrossRef] [PubMed]

24. Nambara, E.; Marion-Poll, A. Abscisic acid biosynthesis and catabolism. Annu. Rev. Plant Biol. 2005, 56, 165-185. [CrossRef]

25. Nishimura, N.; Sarkeshik, A.; Nito, K.; Park, S.; Wang, A.; Carvalho, P.C.; Lee, S.; Caddell, D.F.; Cutler, S.R.; Chory, J.; et al. PYR/PYL/RCAR family members are major in-vivo ABI1 protein phosphatase 2C-interacting proteins in Arabidopsis. Plant $J$. 2010, 61, 290-299. [CrossRef]

26. Udvardi, M.K.; Kakar, K.; Wandrey, M.; Montanari, O.; Murray, J.; Andriankaja, A.; Zhang, J.-Y.; Benedito, V.; Hofer, J.; Chueng, F.; et al. Legume Transcription Factors: Global Regulators of Plant Development and Response to the Environment. Plant Physiol. 2007, 144, 538-549. [CrossRef]

27. Narusaka, Y.; Nakashima, K.; Shinwari, Z.K.; Sakuma, Y.; Furihata, T.; Abe, H.; Narusaka, M.; Shinozaki, K.; Yamaguchi-Shinozaki, K. Interaction between two cis-acting elements, ABRE and DRE, in ABA-dependent expression of Arabidopsis rd29A gene in response to dehydration and high-salinity stresses. Plant J. 2003, 34, 137-148. [CrossRef] [PubMed]

28. Zhang, H.; Cui, X.; Guo, Y.; Luo, C.; Zhang, L. Picea wilsonii transcription factor NAC2 enhanced plant tolerance to abiotic stress and participated in RFCP1-regulated flowering time. Plant Mol. Biol. 2018, 98, 471-493. [CrossRef]

29. Liang, K.-H.; Wang, A.-B.; Yuan, Y.-H.; Miao, Y.-H.; Zhang, L.-Y. Picea wilsonii NAC Transcription Factor PwNAC30 Negatively Regulates Abiotic Stress Tolerance in Transgenic Arabidopsis. Plant Mol. Biol. Rep. 2020, 38, 554-571. [CrossRef]

30. Guo, Y.; Zhang, H.; Yuan, Y.; Cui, X.; Zhang, L. Identification and characterization of NAC genes in response to abiotic stress conditions in Picea wilsonii using transcriptome sequencing. Biotechnol. Biotechnol. Equip. 2020, 34, 93-103. [CrossRef]

31. Li, S.; Zhang, J.; Liu, L.; Wang, Z.; Li, Y.; Guo, L.; Li, Y.; Zhang, X.; Ren, S.; Zhao, B.; et al. SlTLFP8 reduces water loss to improve water-use efficiency by modulating cell size and stomatal density via endoreduplication. Plant Cell Environ. 2020, 43, 2666-2679. [CrossRef]

32. Fujita, M.; Fujita, Y.; Maruyama, K.; Seki, M.; Hiratsu, K.; Ohme-Takagi, M.; Tran, L.-S.P.; Yamaguchi-Shinozaki, K.; Shinozaki, K. A dehydration-induced NAC protein, RD26, is involved in a novel ABA-dependent stress-signaling pathway. Plant J. 2004, 39, 863-876. [CrossRef]

33. Nuruzzaman, M.; Sharoni, A.M.; Kikuchi, S. Roles of NAC transcription factors in the regulation of biotic and abiotic stress responses in plants. Front. Microbiol. 2013, 4, 248. [CrossRef]

34. Baxter, A.; Mittler, R.; Suzuki, N. ROS as key players in plant stress signalling. J. Exp. Bot. 2014, 65, 1229-1240. [CrossRef] [PubMed]

35. Huang, W.; Yang, Y.-J.; Hu, H.; Cao, K.-F.; Zhang, S.-B. Sustained Diurnal Stimulation of Cyclic Electron Flow in Two Tropical Tree Species Erythrophleum guineense and Khaya ivorensis. Front. Plant Sci. 2016, 7, 1068. [CrossRef]

36. Williamson, R.; Field, J.G.; Shillington, F.A.; Jarre, A.; Potgieter, A. A Bayesian approach for estimating vertical chlorophyll profiles from satellite remote sensing: Proof-of-concept. ICES J. Mar. Sci. 2010, 68, 792-799. [CrossRef]

37. Shen, C.; Zhang, Y.; Li, Q.; Liu, S.; He, F.; An, Y.; Zhou, Y.; Liu, C.; Yin, W.; Xia, X. PdGNC confers drought tolerance by mediating stomatal closure resulting from $\mathrm{NO}$ and $\mathrm{H}_{2} \mathrm{O}_{2}$ production via the direct regulation of PdHXK1 expression in Populus. New Phytol. 2021, 230, 1868-1882. [CrossRef]

38. Acebron, K.; Matsubara, S.; Jedmowski, C.; Emin, D.; Muller, O.; Rascher, U. Diurnal dynamics of nonphotochemical quenching in Arabidopsis npq mutants assessed by solar-induced fluorescence and reflectance measurements in the field. New Phytol. 2021, 229, 2104-2119. [CrossRef] [PubMed]

39. Liang, K.; Wang, A.; Sun, Y.; Yu, M.; Zhang, L. Identification and Expression of NAC Transcription Factors of Vaccinium corymbosum L. in Response to Drought Stress. Forests 2019, 10, 1088. [CrossRef]

40. Sun, Y.; Liu, X.; Zhai, H.; Gao, H.; Yao, Y.; Du, Y. Responses of photosystem II photochemistry and the alternative oxidase pathway to heat stress in grape leaves. Acta Physiol. Plant 2016, 38, 232. [CrossRef]

41. Dou, H.; Xv, K.; Meng, Q.; Li, G.; Yang, X. Potato plants ectopically expressing Arabidopsis thaliana CBF3 exhibit enhanced tolerance to high-temperature stress. Plant Cell Environ. 2014, 38, 61-72. [CrossRef]

42. Liu, C.; Sun, Q.; Zhao, L.; Li, Z.; Peng, Z.; Zhang, J. Heterologous Expression of the Transcription Factor EsNAC1 in Arabidopsis Enhances Abiotic Stress Resistance and Retards Growth by Regulating the Expression of Different Target Genes. Front. Plant Sci. 2018, 9, 1495. [CrossRef] [PubMed] 
43. Yang, X.; Wang, X.; Ji, L.; Yi, Z.; Fu, C.; Ran, J.; Hu, R.; Zhou, G. Overexpression of a Miscanthus lutarioriparius NAC gene MINAC5 confers enhanced drought and cold tolerance in Arabidopsis. Plant Cell Rep. 2015, 34, 943-958. [CrossRef] [PubMed]

44. Fang, J.; Chai, Z.; Yao, W.; Chen, B.; Zhang, M. Interactions between ScNAC23 and ScGAI regulate GA-mediated flowering and senescence in sugarcane. Plant Sci. 2020, 304, 110806. [CrossRef]

45. Kovalchuk, N.; Jia, W.; Eini, O.; Morran, S.; Pyvovarenko, T.; Fletcher, S.; Bazanova, N.; Harris, J.; Beck-Oldach, K.; Shavrukov, Y.; et al. Optimization of TaDREB3 gene expression in transgenic barley using cold-inducible promoters. Plant Biotechnol. J. 2013, 11, 659-670. [CrossRef] [PubMed]

46. He, F.; Wang, H.-L.; Li, H.-G.; Su, Y.; Li, S.; Yang, Y.; Feng, C.-H.; Yin, W.; Xia, X. PeCHYR1, a ubiquitin E3 ligase from Populus euphratica, enhances drought tolerance via ABA-induced stomatal closure by ROS production in Populus. Plant Biotechnol. J. 2018, 16, 1514-1528. [CrossRef]

47. Xu, Y.; Zhao, X.; Aiwaili, P.; Mu, X.; Zhao, M.; Zhao, J.; Cheng, L.; Ma, C.; Gao, J.; Hong, B. A zinc finger protein BBX19 interacts with ABF3 to affect drought tolerance negatively in chrysanthemum. Plant J. 2020, 103, 1783-1795. [CrossRef]

48. Sakuraba, Y.; Kim, D.; Han, S.-H.; Kim, S.-H.; Piao, W.; Yanagisawa, S.; An, G.; Paek, N.-C. Multilayered Regulation of MembraneBound ONAC054 is essential for Abscisic Acid-Induced Leaf Senescence in Rice. Plant Cell 2020, 32, 630-649. [CrossRef]

49. Joshi, P.S.; Agarwal, P.; Agarwal, P.K. Overexpression of AlNAC1 from recretohalophyte Aeluropus lagopoides alleviates drought stress in transgenic tobacco. Environ. Exp. Bot. 2021, 181, 104277. [CrossRef]

50. Romero-Romero, J.L.; Inostroza-Blancheteau, C.; Orellana, D.; Aquea, F.; Reyes-Díaz, M.; Gil, P.M.; Matte, J.P.; Arce-Johnson, P. Stomata regulation by tissue-specific expression of the Citrus sinensis MYB61 transcription factor improves water-use efficiency in Arabidopsis. Plant Physiol. Biochem. 2018, 130, 54-60. [CrossRef] [PubMed]

51. Romano, J.M.; Dubos, C.; Prouse, M.B.; Wilkins, O.; Hong, H.; Poole, M.; Kang, K.-Y.; Li, E.; Douglas, C.J.; Western, T.L.; et al. At MYB61, an R2R3-MYB transcription factor, functions as a pleiotropic regulator via a small gene network. New Phytol. 2012, 195, 774-786. [CrossRef]

52. Grondin, A.; Rodrigues, O.; Verdoucq, L.; Merlot, S.; Leonhardt, N.; Maurel, C. Aquaporins Contribute to ABA-Triggered Stomatal Closure through OST1-Mediated Phosphorylation. Plant Cell 2015, 27, 1945-1954. [CrossRef] [PubMed]

53. Ju, Y.-L.; Yue, X.-F.; Min, Z.; Wang, X.-H.; Fang, Y.-L.; Zhang, J.-X. VvNAC17, a novel stress-responsive grapevine (Vitis vinifera L.) NAC transcription factor, increases sensitivity to abscisic acid and enhances salinity, freezing, and drought tolerance in transgenic Arabidopsis. Plant Physiol. Biochem. 2020, 146, 98-111. [CrossRef]

54. Yoshida, T.; Fujita, Y.; Maruyama, K.; Mogami, J.; Todaka, D.; Shinozaki, K.; Yamaguchi-Shinozaki, K. Four A rabidopsis AREB/ABF transcription factors function predominantly in gene expression downstream of SnRK2 kinases in abscisic acid signalling in response to osmotic stress. Plant Cell Environ. 2014, 38, 35-49. [CrossRef]

55. Yoshida, T.; Fujita, Y.; Sayama, H.; Kidokoro, S.; Maruyama, K.; Mizoi, J.; Shinozaki, K.; Yamaguchi-Shinozaki, K. AREB1, AREB2, and ABF3 are master transcription factors that cooperatively regulate ABRE-dependent ABA signaling involved in drought stress tolerance and require ABA for full activation. Plant J. 2010, 61, 672-685. [CrossRef] [PubMed]

56. Chang, H.-C.; Tsai, M.-C.; Wu, S.-S.; Chang, I.-F. Regulation of ABI5 expression by ABF3 during salt stress responses in Arabidopsis thaliana. Bot. Stud. 2019, 60, 1-14. [CrossRef]

57. Yang, Y.; Li, H.-G.; Wang, J.; Wang, H.-L.; He, F.; Su, Y.; Zhang, Y.; Feng, C.-H.; Niu, M.; Li, Z.; et al. ABF3 enhances drought tolerance via promoting ABA-induced stomatal closure by directly regulating ADF5 in Populus euphratica. J. Exp. Bot. 2020, 71, 7270-7285. [CrossRef]

58. Yang, Q.; Yang, B.; Li, J.; Wang, Y.; Tao, R.; Yang, F.; Wu, X.; Yan, X.; Ahmad, M.; Shen, J.; et al. ABA-responsive ABRE-binding factor3 activates DAM3 expression to promote bud dormancy in Asian pear. Plant Cell Environ. 2020, 43, 1360-1375. [CrossRef]

59. Li, X.; Li, X.; Li, M.; Yan, Y.; Liu, X.; Li, L. Dual Function of NAC072 in ABF3-Mediated ABA-Responsive Gene Regulation in Arabidopsis. Front. Plant Sci. 2016, 7, 1075. [CrossRef]

60. Tran, L.-S.P.; Nakashima, K.; Sakuma, Y.; Osakabe, Y.; Qin, F.; Simpson, S.D.; Maruyama, K.; Fujita, Y.; Shinozaki, K.; YamaguchiShinozaki, K. Co-expression of the stress-inducible zinc finger homeodomain ZFHD1 and NAC transcription factors enhances expression of the ERD1 gene in Arabidopsis. Plant J. 2006, 49, 46-63. [CrossRef] [PubMed]

61. Hwang, K.; Susila, H.; Nasim, Z.; Jung, J.-Y.; Ahn, J.H. Arabidopsis ABF3 and ABF4 Transcription Factors Act with the NF-YC Complex to Regulate SOC1 Expression and Mediate Drought-Accelerated Flowering. Mol. Plant 2019, 12, 489-505. [CrossRef] [PubMed]

62. Yamaguchi-Shinozaki, K. A novel cis-acting element in an Arabidopsis gene is involved in responsiveness to drought, lowtemperature, or high-salt stress. Plant Cell 1994, 6, 251-264. [CrossRef] [PubMed]

63. Sun, J.; Peng, X.; Fan, W.; Tang, M.; Liu, J.; Shen, S. Functional analysis of BpDREB2 gene involved in salt and drought response from a woody plant Broussonetia papyrifera. Gene 2014, 535, 140-149. [CrossRef]

64. Tang, M.; Liu, X.; Deng, H.; Shen, S. Over-expression of JcDREB, a putative AP2/EREBP domain-containing transcription factor gene in woody biodiesel plant Jatropha curcas, enhances salt and freezing tolerance in transgenic Arabidopsis thaliana. Plant Sci. 2011, 181, 623-631. [CrossRef] [PubMed]

65. Kim, J.-S.; Mizoi, J.; Yoshida, T.; Fujita, Y.; Nakajima, J.; Ohori, T.; Todaka, D.; Nakashima, K.; Hirayama, T.; Shinozaki, K.; et al. An ABRE Promoter Sequence is Involved in Osmotic Stress-Responsive Expression of the DREB2A Gene, Which Encodes a Transcription Factor Regulating Drought-Inducible Genes in Arabidopsis. Plant Cell Physiol. 2011, 52, 2136-2146. [CrossRef] [PubMed] 
66. Nakashima, K.; Fujita, Y.; Katsura, K.; Maruyama, K.; Narusaka, Y.; Seki, M.; Shinozaki, K.; Yamaguchi-Shinozaki, K. Transcriptional Regulation of ABI3- and ABA-responsive Genes Including RD29B and RD29A in Seeds, Germinating Embryos, and Seedlings of Arabidopsis. Plant Mol. Biol. 2006, 60, 51-68. [CrossRef]

67. Min, M.K.; Kim, R.; Hong, W.-J.; Jung, K.-H.; Lee, J.-Y.; Kim, B.-G. OsPP2C09 Is a Bifunctional Regulator in Both ABA-Dependent and Independent Abiotic Stress Signaling Pathways. Int. J. Mol. Sci. 2021, 22, 393. [CrossRef]

68. Mergby, D.; Hanin, M.; Saidi, M.N. The durum wheat NAC transcription factor TtNAC2A enhances drought stress tolerance in Arabidopsis. Environ. Exp. Bot. 2021, 186, 104439. [CrossRef]

69. Ren, G.; An, K.; Liao, Y.; Zhou, X.; Cao, Y.; Zhao, H.; Ge, X.; Kuai, B. Identification of a Novel Chloroplast Protein AtNYE1 Regulating Chlorophyll Degradation during Leaf Senescence in Arabidopsis. Plant Physiol. 2007, 144, 1429-1441. [CrossRef]

70. Lv, S.; Zhang, Y.; Pan, L.; Liu, Z.; Yang, N.; Pan, L.; Wu, J.; Wang, J.; Yang, J.; Lv, Y.; et al. Strigolactone-triggered stomatal closure requires hydrogen peroxide synthesis and nitric oxide production in an abscisic acid-independent manner. New Phytol. 2018, 217, 290-304. [CrossRef] 